\title{
Equilibrium Investment Strategy for DC Pension Plan with Inflation and Stochastic Income under Heston's SV Model
}

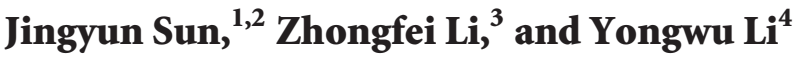 \\ ${ }^{1}$ School of Mathematics, Lanzhou City University, Lanzhou 730070, China \\ ${ }^{2}$ School of Mathematics and Statistics, Lanzhou University, Lanzhou 730000, China \\ ${ }^{3}$ Sun Yat-sen Business School, Sun Yat-sen University, Guangzhou 510275, China \\ ${ }^{4}$ Academy of Mathematics and Systems Science, Chinese Academy of Sciences, Beijing 100190, China \\ Correspondence should be addressed to Zhongfei Li; lnslzf@mail.sysu.edu.cn
}

Received 8 December 2015; Revised 2 April 2016; Accepted 4 April 2016

Academic Editor: Reza Jazar

Copyright ( 2016 Jingyun Sun et al. This is an open access article distributed under the Creative Commons Attribution License, which permits unrestricted use, distribution, and reproduction in any medium, provided the original work is properly cited.

\begin{abstract}
We consider a portfolio selection problem for a defined contribution (DC) pension plan under the mean-variance criteria. We take into account the inflation risk and assume that the salary income process of the pension plan member is stochastic. Furthermore, the financial market consists of a risk-free asset, an inflation-linked bond, and a risky asset with Heston's stochastic volatility (SV). Under the framework of game theory, we derive two extended Hamilton-Jacobi-Bellman (HJB) equations systems and give the corresponding verification theorems in both the periods of accumulation and distribution of the DC pension plan. The explicit expressions of the equilibrium investment strategies, corresponding equilibrium value functions, and the efficient frontiers are also obtained. Finally, some numerical simulations and sensitivity analysis are presented to verify our theoretical results.
\end{abstract}

\section{Introduction}

Nowadays, the application of stochastic control theory to portfolio selection problems of pension funds is becoming a hot issue in actuarial research. The basic pension plans have two types: the defined benefit (DB) pension plan and the defined contribution (DC) pension plan. In recent years, with the rapid development of the equity market and the lower mortality level of the population, compared with the DB pension plan, the DC pension plan is more favored by most countries in the world. As in a DC pension plan, the payment pressure of benefit which is caused by the uncertainty of investment earnings and pension plan member's longevity risk is transferred from the sponsoring company to the member himself/herself. Thus, in the literature related to pension funds, the majority of the literature focuses on the DC pension plan.

As we know, for a DC pension plan, the contribution is a predetermined constant or a fixed proportion of the member's income while the benefit is distributed based on the accumulation value of the contribution and the return of the pension fund portfolio until retirement. Thus, many scholars are devoted to the optimal investment problem of the DC pension plan. For example, [1] considered a discretetime multiperiod DC pension plan model to minimize the expected deviation between the pension fund account and a predetermined target by using a quadratic loss function; to maximize the expected utility from the wealth at retirement time, [2] investigated the optimal investment strategies for a DC pension plan both before and after retirement under the continuous-time framework; [3] extended the above model into the case with stochastic interest rate and stochastic labor income; [4] obtained the closed-form of the optimal investment strategy for a DC pension plan under the logarithm utility function. Besides, [5] investigated the optimal asset allocation problem for a DC pension plan with downside protection under stochastic inflation, and [6] studied the same problem under the stochastic interest rate and stochastic volatility framework. Under the regime switching environment, [7] considered an optimal assetliability management problem for a pension fund.

All the literature mentioned above focus on the optimal investment strategy under the objective of maximizing the expected utility or minimizing the expected quadratic 
loss. In recent years, some scholars pay attention to the portfolio selection problem of the DC pension plan under the mean-variance (MV) criteria. This is because of the fact that the optimal investment problems under the MV criteria in a multiperiod or continuous-time framework are successfully solved only recently. It is well known that the MV problems with multiperiod or continuous-time version are time-inconsistent in the sense that the Bellman optimality principle does not hold. Hence, the dynamic programming approach can not be used. In existing literature, there are usually two methods that are suggested to deal with this problem. The first one is to find the precommitment strategy (the breakthrough work for this method was made by $[8,9]$ ), which means that the manager derives an optimal strategy at the initial time 0 and commits to performing this strategy in the future, even if it does not remain optimal in the later time. However, in practice, this strategy is not easily performed, since the preference of the MV manager changes with time and he/she has an incentive to deviate from the precommitment strategy in the later time. In addition, finding a time-consistent strategy is a basic requirement for a rational decision-maker. Thus, the second method is finding a timeconsistent equilibrium strategy under the framework of game theory (the most representative work was made by [10-12]). In this case, we regard the decision-making process as a noncooperative game between an infinite number of distinct players. At each time $t$, we named player $t$, representing the future incarnation of the manager at time $t$. We are interested in finding a subgame perfect Nash equilibrium point for the game and formulating an equilibrium strategy; this strategy is time-consistent. For the MV problem under the background of pension fund, [13] considered a DC pension plan with the return of premiums clauses under the game theoretic framework and obtained an equilibrium investment strategy in the period before retirement. Under the same framework, [14] investigated an equilibrium investment and contribution strategies for a DB pension plan. The research on the precommitment investment strategy can be found in [15-17] and references therein.

Meanwhile, for the portfolio problem, many scholars begin to pay attention to the influence of the background risk, such as the risk of interest rate, inflation, and volatility of the risky asset, on the optimal decision-making process. On the one hand, as we all know, the inflation might affect the real value of the wealth; especially for the problem with long time horizon investment, the higher inflation leads to the lower real wealth. In the DC pension plan, [18] obtained the optimal investment strategy which maximizes the expected utility of the DC pension plan under inflation risk. A similar problem was studied in [5] under the environment of stochastic interest and inflation rate with the minimized guarantee. Under the MV criteria, [17] investigated the optimal precommitment investment strategy of a DC pension plan with inflation risk using Lagrange method. Recently, the equilibrium investment strategies for a DC pension plan with the inflation risk are obtained by [19]. On the other hand, many empirical studies have shown that the volatility of the risky asset is stochastic. Some scholars have proposed a variety of SV models, such as the constant elastic variance (CEV) model
[20], Heston's SV model (volatility satisfies Cox-IngersollRoss process) [21], and Stein-Stein model (volatility satisfies Ornstein-Uhlenbeck process) [22]. The CEV and Heston's SV models have been widely considered in investment and reinsurance problems such as [23-25] and references therein. For the stochastic volatility model under the DC pension plan, the interested reader can be referred to $[6,26,27]$.

In this paper, we consider the MV portfolio problems for a DC pension plan both before and after retirement. The main difference between this paper and the existing literature is that both the inflation risk and the stochastic volatility risk are considered in our model. To the best of our knowledge, under the MV framework, there is no literature considering both of the two risks in the DC pension fund management. We assume that the financial market consists of a risk-free asset, an inflation-linked bond, and a stock with Heston's stochastic volatility. The salary income of the DC pension plan member is also assumed to be stochastic because of the influence of inflation rate. Under the framework of game theory, two MV problems are formulated related to the periods before and after retirement, respectively. For each of the problems, by solving an extended HJB equations system, the equilibrium strategy, equilibrium value function, and the corresponding equilibrium efficient frontier are obtained. We find that the equilibrium investment money in the inflationlinked bond depends on current wealth; the inflation risk and the contribution of the salary income have no influence on the equilibrium investment money of stock. Finally, using Monte Carlo method, we investigate the evolution process of the equilibrium strategy with time before retirement under different parameters and present the sensitivity of the efficient frontier to corresponding parameters.

The remainder of the paper is organized as follows. In Section 2, we introduce the financial market and wealth processes both before and after retirement. In Section 3, a MV portfolio allocation problem is formulated in the period before retirement. Under the framework of game theory, an extended HJB equations system and an equilibrium investment strategy are also obtained. In Section 4, we consider a MV portfolio problem after retirement and derive the corresponding verification theorem and the equilibrium strategy. Some numerical simulations and sensitivity analysis for our results are presented in Section 5. Section 6 concludes the paper and outlines further research.

\section{Assumption and Model}

Let $(\Omega, \mathscr{F}, \mathbb{F}, \mathbb{P})$ be a filtered probability space with filtration $\mathbb{F}=\left\{\mathscr{F}_{t}\right\}_{t \in[0, T+N]}$ satisfying the usual conditions; that is, $\left\{\mathscr{F}_{t}\right\}_{t \in[0, T+N]}$ is right-continuous and $\mathbb{P}$-complete. The time horizon $[0, T]$ represents the accumulation period of a DC pension plan member, and $[T, T+N]$ is the distribution period of the member after he/she retires. Let $\mathscr{F}_{t}$ represent the information available until time $t$. Suppose that all of stochastic processes and random variables are defined on the filtered probability space $(\Omega, \mathscr{F}, \mathbb{F}, \mathbb{P})$. In addition, we assume that there are no transaction costs or taxes in the financial market, trading can take place continuously, and short selling is permitted. 
2.1. Financial Market. As we all know, for a DC pension plan manager, the objective is making the terminal wealth maximized by investing the pension fund into the market. Generally speaking, the investment time horizon of the pension fund lasts decades. The inflation risk, as a kind of important background risk, has important influence on the real value of the pension fund. In economics, CPI (Consumer Price Index) as the typical index can represent the inflation level of the market. Following [28, 29], we assume that the price level $P(t)$ satisfies the following diffusion process:

$$
\frac{d P(t)}{P(t)}=\mu_{p}(t) d t+\sigma_{p} d W_{p}(t), \quad P(0)=p_{0},
$$

where $\mu_{p}(t)$ represents the instantaneous expected inflation rate, $\sigma_{p}>0$ is the instantaneous volatility of inflation rate, and $W_{p}(t)$ is a standard Brownian motion, which generates uncertainty of the price level.

We assume that the financial market consists of three kinds of assets: an inflation-linked asset, a money market account, and a stock.

(1) The inflation-linked index bond has the same risk source as the price level process $P(t)$ and can be freely traded in the market. Following $[18,29]$, its price process $I(t)$ satisfies the following stochastic differential equation (SDE):

$$
\begin{aligned}
\frac{d I(t)}{I(t)} & =r(t) d t+\frac{d P(t)}{P(t)} \\
& =\left(r(t)+\mu_{p}(t)\right) d t+\sigma_{p} d W_{p}(t),
\end{aligned}
$$

where $r(t)$ represents the real interest rate at time $t . r(t)+\mu_{p}(t)$ is the expected yields of the inflation-linked bond. The higher expected inflation rate $\mu_{p}(t)$ will lead to higher expected yields of the inflation-linked bond. Thus, it can hedge the inflation risk.

(2) The price dynamics of the risk-free money market account is given by

$$
\frac{d B(t)}{B(t)}=R(t) d t
$$

where $R(t)$ is the nominal interest rate.

(3) The risky asset in the market is a stock, whose price process $S(t)$ follows a geometric Brownian motion with Heston's stochastic volatility. That is,

$$
\begin{aligned}
& \frac{d S(t)}{S(t)}= R(t) d t+\sqrt{V(t)}\left(\lambda_{s} \sqrt{V(t)} d t+d W_{s}(t)\right) \\
&+\sigma_{s}\left(\lambda_{p}(t) d t+d W_{p}(t)\right) \\
&= {\left[R(t)+\lambda_{s} V(t)+\lambda_{p}(t) \sigma_{s}\right] d t } \\
&+\sqrt{V(t)} d W_{s}(t)+\sigma_{s} d W_{p}(t), \\
& d V(t)= \alpha[\delta-V(t)] d t+\sigma_{v} \sqrt{V(t)} d W_{v}(t), \\
& \quad V(0)=v_{0},
\end{aligned}
$$

where $W_{s}(t)$ and $W_{v}(t)$ are two standard Brownian motions and $\lambda_{s} \sqrt{V(t)}$ and $\lambda_{p}(t)$ are the market price of the risk sources
$W_{s}(t)$ and $W_{p}(t)$, respectively. Since the inflation risk might have influence on the price evolution process of the stock through direct or indirect ways (the correlated empirical research can be found in $[30,31]$ and references therein), hence, we assume that the price process of the stock is derived by not only its own risk source $W_{s}(t)$, but also the risk source $W_{p}(t)$. We further assume that $W_{p}(t)$ is independent of $W_{s}(t)$ and $W_{v}(t)$, respectively, while $W_{s}(t)$ and $W_{v}(t)$ are dependent, and $\mathbb{E}\left[W_{s}(t) W_{v}(t)\right]=\rho_{s v} t$, where $\rho_{s v} \in[-1,1]$ is the correlation coefficient. The second equation of (4) is a CIR (Cox-Ingersoll-Ross) mean-revert process, which models the stochastic volatility of the stock price. Here we suppose $2 \alpha \delta>$ $\sigma_{v}^{2}$ to assure that $V(t)>0$ holds.

In addition, we assume that the DC pension plan member receives salary income with nominal value $\bar{L}(t)$ at time $t$ until the retirement time $T$. Suppose that the income is stochastic and dynamically influenced by the price level $P(t)$; that is, the salary income process is driven by the source of uncertainty from inflation, and it satisfies the following process:

$$
\begin{aligned}
\frac{d \bar{L}(t)}{\bar{L}(t)} & =\mu_{l} d t+\sigma_{l} d W_{p}(t), \\
\bar{L}(0) & =\bar{l}_{0}>0,
\end{aligned}
$$

where $\mu_{l}$ is the average growth rate of the income and $\sigma_{l}$ is the volatility rate.

Remark 1 . To simplify the model, achieve tractability, and give detailed analysis about the optimal strategies, we assume that the real interest rate $r(t)$, nominal interest rate $R(t)$, and expected inflation rate $\mu_{p}(t)$ are all deterministic functions of time $t$.

Remark 2. If we denote $\mathbb{Q}$ as the risk neutral measure, then (2) can be rewritten as

$$
\frac{d I(t)}{I(t)}=\left(r(t)+\mu_{p}(t)-\lambda_{p}(t) \sigma_{p}\right) d t+\sigma_{p} d W_{p}^{\mathbb{Q}}(t) .
$$

By the pricing theory of the derivative (to avoid arbitrage), we obtain the following relationship:

$$
r(t)+\mu_{p}(t)-\lambda_{p}(t) \sigma_{p}=R(t) .
$$

Remark 3. In the empirical research, the evolution process of $S(t)$ and $V(t)$ is usually negatively correlated. Since in general, with declining of the stock price, the volatility of the price gradually increases (cf. [32]), based on this reason, in Section 5, we assume that the parameter $\rho_{s v}<0$.

2.2. Wealth Process. In this paper, we consider the optimal portfolio problems of a DC pension plan member both before and after retirement. The member contributes part of his/her salary into the pension fund account before retirement and obtains benefit after retirement. So we need to consider the wealth processes in two different periods.

(I) Before Retirement. During the accumulation period $[0, T]$, we assume that the DC pension plan member contributes 
continuously to his/her pension fund account with a fixed rate $k$ of his/her salary income; that is, he/she contributes the amount of money $k \bar{L}(t)$ to his/her pension fund account at time $t$. The pension fund is invested in the financial market by the manager. Denote $\pi_{I}(t)$ and $\pi_{S}(t)$ as the propositions of wealth invested in the inflation-linked index bond and the stock, respectively. Then $\pi_{B}(t)=1-\pi_{I}(t)-\pi_{S}(t)$ is the proposition of the risk-free money market account. Denote $\pi(t)=\left(\pi_{I}(t), \pi_{S}(t)\right), t \in[0, T]$, as the decision-making process. Now we rewrite the SDEs (2) and (4) as matrix form:

$$
\begin{aligned}
& {\left[\begin{array}{c}
\frac{d I(t)}{I(t)} \\
\frac{d S(t)}{S(t)}
\end{array}\right]=} \underbrace{\left[\begin{array}{c}
r(t)+\mu_{p}(t) \\
R(t)+\lambda_{s} V(t)+\lambda_{p}(t) \sigma_{s}
\end{array}\right]}_{\mu(t)} d t \\
&+\underbrace{\left[\begin{array}{cc}
\sigma_{p} & 0 \\
\sigma_{s} & \sqrt{V(t)}
\end{array}\right]}_{\Sigma(t)} \underbrace{\left[\begin{array}{l}
d W_{p}(t) \\
d W_{s}(t)
\end{array}\right]}_{d W(t)} .
\end{aligned}
$$

Denote $\bar{X}^{\pi}(t)$ as the nominal value of the wealth process associated with the investment strategy $\pi$. Then the nominal wealth process $\bar{X}^{\pi}(t)$ satisfies

$$
\begin{aligned}
d \bar{X}^{\pi}(t) & \\
= & \bar{X}^{\pi}(t)\left[\pi_{B}(t) \frac{d B(t)}{B(t)}+\pi_{I}(t) \frac{d I(t)}{I(t)}+\pi_{S}(t) \frac{d S(t)}{S(t)}\right] \\
& +k \bar{L}(t) d t \\
= & \bar{X}^{\pi}(t)[R(t)+\pi(t)(\mu(t)-R(t) \mathbf{1})] d t \\
& +\bar{X}^{\pi}(t) \pi(t) \Sigma(t) d W(t)+k \bar{L}(t) d t \\
= & \bar{X}^{\pi}(t)[R(t)+\pi(t) \Sigma(t) \theta(t)] d t+k \bar{L}(t) d t \\
& +\bar{X}^{\pi}(t) \pi(t) \Sigma(t) d W(t),
\end{aligned}
$$

where $\mathbf{1}=(1,1)^{\prime}$. The last equation holds because of (7) and

$$
\begin{aligned}
\mu(t)-R(t) \mathbf{1} & =\left[\begin{array}{c}
r(t)-R(t)+\mu_{p}(t) \\
\lambda_{s} V(t)+\lambda_{p}(t) \sigma_{s}
\end{array}\right] \\
& =\left[\begin{array}{c}
\lambda_{p}(t) \sigma_{p} \\
\lambda_{s} V(t)+\lambda_{p}(t) \sigma_{s}
\end{array}\right]=\Sigma(t) \theta(t),
\end{aligned}
$$

where $\theta(t)=\left(\lambda_{p}(t), \lambda_{s} \sqrt{V(t)}\right)^{\prime}$. Let $\bar{x}_{0}$ be the nominal wealth at time 0 .

Since the real value of the wealth reflects the real purchase power of the current market, the nominal value of the wealth should be converted into the real value. By discounting the value of nominal wealth with the price level process, the real value of wealth will be obtained. Now denote $X(t)=$ $\bar{X}(t) / P(t)$ and $L(t)=\bar{L}(t) / P(t)$, and, by Itô's formula, we obtain the real wealth process $X^{\pi}(t)$ and real salary income process $L(t)$ as follows:

$$
\begin{aligned}
& d X^{\pi}(t)=X^{\pi}(t) \\
& \cdot\left[R(t)-\mu_{p}(t)+\sigma_{p}^{2}+\pi(t) \Sigma(t)\left(\theta(t)-\rho_{p}\right)\right] d t \\
& \quad+k L(t) d t+X^{\pi}(t)\left(\pi(t) \Sigma(t)-\rho_{p}^{\prime}\right) d W(t), \\
& d L(t)=L(t)\left[\alpha_{l}(t) d t+\beta_{l}^{\prime} d W(t)\right],
\end{aligned}
$$

where $\alpha_{l}(t)=\mu_{l}-\mu_{p}(t)+\sigma_{p}^{2}-\sigma_{l} \sigma_{p}$ and $\rho_{p}^{\prime}=\left(\sigma_{p}, 0\right)$, $\beta_{l}^{\prime}=\left(\sigma_{l}-\sigma_{p}, 0\right)$. For $P(0)=p_{0}$, the real initial wealth and initial salary income are $X^{\pi}(0)=\bar{x}_{0} / p_{0}$ and $L(0)=\bar{l}_{0} / p_{0}$, respectively. Note that the dynamic process of $X^{\pi}(t)$ depends on the real salary income process $L(t)$ and the stochastic volatility process $V(t)$, but it is independent of the price level process $P(t)$.

Definition 4 (admissible strategy). Let $\mathcal{O}:=\mathbb{R} \times \mathbb{R}^{+} \times \mathbb{R}^{+}$and $\mathscr{G}:=[0, T] \times \mathcal{O}$. A strategy $\pi(t)=\left\{\pi_{I}(t), \pi_{S}(t)\right\}_{t \in[0, T]}$ is said to be admissible if

(1) $\forall(x, v, l) \in \mathcal{O}$, the $\operatorname{SDE}$ (11) has unique solution $\left\{X^{\pi}(s)\right\}_{s \in[t, T]}$ with $X^{\pi}(t)=x, V(t)=v$, and $L(t)=l$;

(2) $\forall s \in[t, T], \mathbb{E}\left[\int_{t}^{T}\left[\left(\pi_{I}(s)-1\right) \sigma_{p}+\pi_{S}(s) \sigma_{s}\right]^{4} d s\right]<\infty$;

(3) $\forall \varrho \in[1,+\infty)$ and $\forall(t, x, v, l) \in \mathscr{G}$, $\mathbb{E}_{t, x, v, l}\left[\sup _{s \in[t, T]}\left|X^{\pi}(s)\right|^{\varrho}\right]<\infty$, where $\mathbb{E}_{t, x, v, l}[\cdot]$ is the condition expectation given $X^{\pi}(t)=x, V(t)=v$, and $L(t)=l$.

One denotes $\Pi(t, x, v, l)$ as the set of all admissible strategies with respect to the initial condition $(t, x, v, l) \in \mathscr{G}$.

(II) After Retirement. When the member of the pension plan retires, the accumulation value of the pension fund usually is used to purchase an annuity. In practice, the member usually has two ways to purchase the annuity. The first way is that he/she purchases the annuity directly at the retirement time. For example, as being considered in [2,27], the accumulated pension fund is used to purchase a paid-up annuity once the member arrives at the retirement age, and the manager has also to decide the part of the remaining mathematical reserve to invest in the market. The second way is that the member does not purchase the annuity directly but chooses the income drawdown option at the retirement. It means that the member withdraws a fixed income and invests the rest of the wealth until he/she achieves the age when the time of purchasing the annuity is compulsory. The interested reader can see $[33,34]$ for details.

Here we consider the first way. Similar to [2], we assume that the member purchases a paid-up annuity at the retirement time which guarantees the benefit to be given only on a fixed time horizon $[T, T+N]$ and invests the rest of the wealth continuously in the market. We denote $W$ as the part of the fund used to purchase an annuity $(W \leqslant X(T))$. Continuous benefit (real value) paid from $T$ to $T_{1}=T+N$ is $q=W / \bar{a}_{\bar{N} \mid}$, where $\bar{a}_{\bar{N} \mid}=\left(1-e^{-\delta N}\right) / \delta$ and $\delta$ is the continuous technical rate. 
Let $M(t)$ denote the nominal total payment value of the benefit at time interval $[T, t], t \in\left(T, T_{1}\right]$. For simplicity, the dynamics of $M(t)$ can be modeled as $M(t)=\int_{T}^{t} q P(s) d s$ or $d M(t)=q P(t) d t$. This setting is reasonable and realistic, and a similar setting is used in [17]. It means that after retirement the nominal benefit is adjusted dynamically based on the inflation rate of that time; that is, at time $t$, the nominal benefit is $q P(t)$, which keeps the purchasing power level of the retirees not decreasing. We assume that once the retired member dies during $\left[T, T_{1}\right]$, the pension fund continues to be invested and the remaining annuity benefit and the terminal wealth $X\left(T_{1}\right)$ are paid off to his/her offspring. If the member is alive until $T_{1}$, the wealth $X\left(T_{1}\right)$ is left for his/her later life. Let $\widehat{\pi}_{I}(t)$ and $\hat{\pi}_{S}(t)$ represent the propositions of the pension fund wealth invested in the inflation-linked index bond and the stock, respectively. Denote $\widehat{\pi}(t)=\left(\widehat{\pi}_{I}(t), \widehat{\pi}_{S}(t)\right), t \in\left[T, T_{1}\right]$, as the decision-making process. Then, after retirement, the dynamics of nominal wealth process is

$$
\begin{aligned}
d \bar{X}^{\hat{\pi}}(t)= & \bar{X}^{\hat{\pi}}(t)[R(t)+\hat{\pi}(t) \Sigma(t) \theta(t)] d t \\
& -q P(t) d t+\bar{X}^{\hat{\pi}}(t) \hat{\pi}(t) \Sigma(t) d W(t) .
\end{aligned}
$$

Using the same method as before retirement, we obtain the real wealth process as follows:

$$
\begin{aligned}
& d X^{\widehat{\pi}}(t)=X^{\widehat{\pi}}(t) \\
& \cdot\left[R(t)-\mu_{p}(t)+\sigma_{p}^{2}+\widehat{\pi}(t) \Sigma(t)\left(\theta(t)-\rho_{p}\right)\right] d t \\
& -q d t+X^{\widehat{\pi}}(t)\left(\widehat{\pi}(t) \Sigma(t)-\rho_{p}^{\prime}\right) d W(t) .
\end{aligned}
$$

Now we give the definition of the admissible strategy for the decision-making process $\widehat{\pi}(t)$.

Definition 5 (admissible strategy). Let $\mathcal{O}_{1}:=\mathbb{R} \times \mathbb{R}^{+}$and $\mathscr{G}_{1}:=\left[T, T_{1}\right] \times \mathcal{O}_{1}$. A strategy $\widehat{\pi}(t)=\left\{\hat{\pi}_{I}(t), \hat{\pi}_{S}(t)\right\}_{t \in\left[T, T_{1}\right]}$ is said to be admissible if

(1) $\forall(x, v) \in \mathcal{O}_{1}$, the $\operatorname{SDE}$ (14) has unique solution $\left\{X^{\widehat{\pi}}(s)\right\}_{s \in\left[t, T_{1}\right]}$ with $X^{\widehat{\pi}}(t)=x, V(t)=v ;$

(2) $\forall s \in\left[t, T_{1}\right], \mathbb{E}\left[\int_{t}^{T_{1}}\left[\left(\widehat{\pi}_{I}(s)-1\right) \sigma_{p}+\widehat{\pi}_{S}(s) \sigma_{s}\right]^{4} d s\right]<\infty$;

(3) $\forall \varrho \in[1,+\infty)$ and $\forall(t, x, v) \in \mathscr{G}_{1}$, $\mathbb{E}_{t, x, v}\left[\sup _{s \in\left[t, T_{1}\right]}\left|X^{\hat{\pi}}(s)\right|^{\varrho}\right]<\infty$, where $\mathbb{E}_{t, x, v}[\cdot]$ is the condition expectation given $X^{\widehat{r}}(t)=x$ and $V(t)=v$.

One denotes $\widehat{\Pi}(t, x, v)$ as the set of all admissible strategies with respect to the initial condition $(t, x, v) \in \mathscr{G}_{1}$.

\section{Problem Formulation before Retirement and Verification Theorem}

In this section, we assume that the pension plan manager hopes to maximize the expectation of the total wealth at retirement time $T$, meanwhile minimizing the variance of the wealth at retirement. So the pension plan manager faces a continuous-time MV portfolio problem. However, since the MV objective function does not satisfy the iterated expectation property, Bellman's optimality principle does not hold under the continuous-time framework. Hence, this problem is time-inconsistent. This means that for the pension fund manager without precommitment should take into account that he/she may have different objective functions in different times. We can thus view this problem as a noncooperative game. At each time $t$, there is one player, named player $t$, representing the future incarnation of the manager at time $t$. At any state $(t, x, v, l)$, the manager faces an optimal control problem with value function $V(t, x, v, l)$ as follows:

$$
\begin{aligned}
J(t, x, v, l, \pi)= & \mathbb{E}_{t, x, v, l}\left[X^{\pi}(T)\right] \\
& -\frac{\gamma}{2} \operatorname{Var}_{t, x, v, l}\left[X^{\pi}(T)\right], \\
V(t, x, v, l)= & \sup _{\pi \in \Pi(t, x, v, l)} J(t, x, v, l, \pi),
\end{aligned}
$$

where $\gamma$ represents the risk aversion level of the manager and $\operatorname{Var}_{t, x, v, l}[\cdot]$ refers to the conditional variance. Since the objective of the pension manager updates with different state, the manager's decision process is like a game process between an infinite number of distinct players. Thus we need to look for a subgame perfect Nash equilibrium point for the game and determine an equilibrium strategy $\pi^{*}$.

If we denote $y^{\pi}(t, x, v, l)=\mathbb{E}_{t, x, v, l}\left[X^{\pi}(T)\right], z^{\pi}(t, x, v, l)=$ $\mathbb{E}_{t, x, v, l}\left[\left(X^{\pi}(T)\right)^{2}\right]$, then the value function $V(t, x, v, l)$ can be rewritten as

$$
\begin{aligned}
V & (t, x, v, l) \\
& =\sup _{\pi \in \Pi(t, x, v, l)}\left\{f\left(t, x, v, l, y^{\pi}(t, x, v, l), z^{\pi}(t, x, v, l)\right)\right\},
\end{aligned}
$$

where $f(t, x, v, l, y, z)=y-(\gamma / 2)\left(z-y^{2}\right)$.

3.1. Verification Theorem. Based on the idea of the Nash equilibrium, similarly to [11], we first give the definition of equilibrium strategy.

Definition 6. For any given initial state $(t, x, v, l) \in \mathscr{G}$, consider an admissible strategy $\pi^{*}(t, x, v, l)$ and choose three real numbers $\tau>0, \tilde{\pi}_{I}$, and $\tilde{\pi}_{B}$; one defines the following strategy:

$$
\begin{aligned}
& \pi_{\tau}(s, \tilde{x}, \tilde{v}, \tilde{l}) \\
& \quad= \begin{cases}\left(\widetilde{\pi}_{I}, \tilde{\pi}_{B}\right), & t \leqslant s<t+\tau,(\tilde{x}, \widetilde{v}, \widetilde{l}) \in \mathcal{O}, \\
\pi^{*}(s, \tilde{x}, \widetilde{v}, \widetilde{l}), & t+\tau \leqslant s<T,(\tilde{x}, \widetilde{v}, \widetilde{l}) \in \mathcal{O} .\end{cases}
\end{aligned}
$$

If $\lim _{\tau \rightarrow 0} \inf \left(\left(f^{\pi^{*}}(t)-f^{\pi_{\tau}}(t)\right) / \tau\right) \geqslant 0 \forall\left(\widetilde{\pi}_{I}, \tilde{\pi}_{B}\right) \in \mathbb{R} \times \mathbb{R}$, then $\pi^{*}(t, x, v, l)$ is called an equilibrium strategy, and the equilibrium value function is defined by

$$
\begin{aligned}
V(t, x, v, l) \\
\quad=f\left(t, x, v, l, y^{\pi^{*}}(t, x, v, l), z^{\pi^{*}}(t, x, v, l)\right),
\end{aligned}
$$

where $f^{\pi}(t)=f\left(t, x, v, l, y^{\pi}(t, x, v, l), z^{\pi}(t, x, v, l)\right)$ for short. 
For any $\phi(t, x, v, l) \in C^{1,2,2,2}(\mathscr{G})$, we define a set $D_{p}(\mathscr{G})=$ $\left\{\phi(t, x, v, l) \mid \phi(t, x, v, l) \in C^{1,2,2,2}(\mathscr{G})\right.$ and all first-order partial derivatives of $\phi(\cdot, x, v, l)$ satisfy the polynomial growth condition on $\mathcal{O}\}$, and we define a variational operator as follows (in the following we will omit the variable $t$ for notation convenience):

$$
\begin{aligned}
\mathscr{A}^{\pi} \phi( & t, x, v, l) \\
= & \phi_{t}+\phi_{x}\left[k l+x\left(R-\mu_{p}+\sigma_{p}^{2}+\pi \Sigma\left(\theta-\rho_{p}\right)\right)\right] \\
& +\phi_{v} \alpha(\delta-v)+\phi_{l} \alpha_{l} l \\
& +\frac{1}{2} \phi_{x x} x^{2}\left(\pi \Sigma-\rho_{p}^{\prime}\right)\left(\pi \Sigma-\rho_{p}^{\prime}\right)^{\prime}+\frac{1}{2} \phi_{v v} \sigma_{v}^{2} v \\
& +\frac{1}{2} \phi_{l l} l^{2} \beta_{l}^{\prime} \beta_{l}+\phi_{x v} x \sigma_{v} \sqrt{v}\left(\pi \Sigma-\rho_{p}^{\prime}\right) \beta_{v} \\
& +\phi_{x l} x l\left(\pi \Sigma-\rho_{p}^{\prime}\right) \beta_{l}+\phi_{v l} l \sigma_{v} \sqrt{v} \beta_{l}^{\prime} \beta_{v},
\end{aligned}
$$

where $\beta_{v}=\left(0, \rho_{s v}\right)^{\prime}$. Now we give the verification theorem of problem $\left(\mathscr{P}_{1}\right)$.

Theorem 7 (verification theorem). For problem $\left(\mathscr{P}_{1}\right)$, if there exist three real value functions $F(t, x, v, l), G(t, x, v, l)$, and $H(t, x, v, l) \in D_{p}(\mathscr{G})$ satisfying the following conditions: $\forall(t, x, v, l) \in \mathscr{G}$,

$$
\begin{aligned}
& \sup _{\pi \in \Pi(t, x, v, l)}\left\{\mathscr{A}^{\pi} F(t, x, v, l)-\xi^{\pi}(t, x, v, l)\right\}=0, \\
& F(T, x, v, l)=f\left(T, x, v, l, x, x^{2}\right), \\
& \mathscr{A}^{\pi^{*}} G(t, x, v, l)=0, \\
& G(T, x, v, l)=x ; \\
& \mathscr{A}^{\pi^{*}} H(t, x, v, l)=0, \\
& H(T, x, v, l)=x^{2},
\end{aligned}
$$

where $\pi^{*}=\arg \sup _{\pi \in \Pi(t, x, v, l)}\left\{\mathscr{A}^{\pi} F(t, x, v, l)-\xi^{\pi}(t, x, v, l)\right\}$, then $V(t, x, v, l)=F(t, x, v, l), y^{\pi^{*}}(t, x, v, l)=G(t, x, v, l)$, and $z^{\pi^{*}}(t, x, v, l)=H(t, x, v, l)$, and $\pi^{*}$ is the equilibrium strategy, where

$$
\begin{aligned}
\xi^{\pi}( & t, x, v, l) \\
= & f_{t}+\left[x\left(R-\mu_{p}+\sigma_{p}^{2}+\pi \Sigma\left(\theta-\rho_{p}\right)\right)+k l\right] f_{x} \\
& +[\alpha(\delta-v)] f_{v}+\alpha_{l} l f_{l} \\
& +\frac{1}{2}\left(\pi \Sigma-\rho_{p}^{\prime}\right)\left(\pi \Sigma-\rho_{p}^{\prime}\right)^{\prime} x^{2} U_{1}^{\pi}+\frac{1}{2} \sigma_{v}^{2} v U_{2}^{\pi} \\
& +\frac{1}{2} l^{2} \beta_{l}^{\prime} \beta_{l} U_{3}^{\pi}+\sigma_{v} \sqrt{v} x\left(\pi \Sigma-\rho_{p}^{\prime}\right) \beta_{v} U_{4}^{\pi} \\
& +x l\left(\pi \Sigma-\rho_{p}^{\prime}\right) \beta_{l} U_{5}^{\pi}+\sigma_{v} \sqrt{v} l \beta_{l}^{\prime} \beta_{v} U_{6}^{\pi},
\end{aligned}
$$

$$
\begin{aligned}
U_{1}^{\pi} & \\
= & f_{x x}+f_{y y}\left(y_{x}^{\pi}\right)^{2}+f_{z z}\left(z_{x}^{\pi}\right)^{2}+2 f_{x y} y_{x}^{\pi}+2 f_{x z} z_{x}^{\pi} \\
& +2 f_{y z} y_{x}^{\pi} z_{x}^{\pi},
\end{aligned}
$$

and $f=f(t, x, v, l, y, z), y^{\pi}=y^{\pi}(t, x, v, l)$, and $z^{\pi}=$ $z^{\pi}(t, x, v, l)$.

The proof of this theorem is given in the Appendix.

Equations (19), (21), and (22) are called an extended HJB equations system. By solving this equations system, we can obtain the equilibrium strategy and corresponding equilibrium value function.

3.2. Solution to the Equilibrium Strategy. Since $f(t, x, v, l, y$, $z)=y-(\gamma / 2)\left(z-y^{2}\right)$, according to Theorem 7 , we have $U_{1}^{\pi^{*}}=\gamma G_{x}^{2}, U_{2}^{\pi^{*}}=\gamma G_{v}^{2}, U_{3}^{\pi^{*}}=\gamma G_{l}^{2}, U_{4}^{\pi^{*}}=\gamma G_{x} G_{v}, U_{5}^{\pi^{*}}=$ $\gamma G_{x} G_{l}$, and $U_{6}^{\pi^{*}}=\gamma G_{v} G_{l}$, so

$$
\begin{aligned}
\xi^{\pi^{*}}(t, x, v, l)= & \frac{1}{2}\left(\pi \Sigma-\rho_{p}^{\prime}\right)\left(\pi \Sigma-\rho_{p}^{\prime}\right)^{\prime} x^{2} \gamma G_{x}^{2} \\
& +\frac{1}{2} \sigma_{v}^{2} v \gamma G_{v}^{2}+\frac{1}{2} l^{2} \beta_{l}^{\prime} \beta_{l} \gamma G_{l}^{2} \\
& +\sigma_{v} \sqrt{v} l \beta_{l}^{\prime} \beta_{v} \gamma G_{v} G_{l} \\
& +\sigma_{v} \sqrt{v} x\left(\pi \Sigma-\rho_{p}^{\prime}\right) \beta_{v} \gamma G_{v} G_{x} \\
& +x l\left(\pi \Sigma-\rho_{p}^{\prime}\right) \beta_{l} \gamma G_{x} G_{l} .
\end{aligned}
$$


By differentiating $\mathscr{A}^{\pi} F(t, x, v, l)-\xi^{\pi}(t, x, v, l)$ with respect to $\pi(t)$, we can obtain the equilibrium strategy

$$
\pi^{*}(t)=-\frac{\left[F_{x}\left(\theta-\rho_{p}\right)^{\prime}+\left(F_{x v}-\gamma G_{x} G_{v}\right) \sigma_{v} \sqrt{v} \beta_{v}^{\prime}+\left(F_{x l}-\gamma G_{x} G_{l}\right) l \beta_{l}^{\prime}\right] \Sigma^{-1}}{\left(F_{x x}-\gamma G_{x}^{2}\right) x}+\rho_{p}^{\prime} \Sigma^{-1}
$$

Inspired by [24], we suppose $F(t, x, v, l)$ and $G(t, x, v, l)$ have the following linear forms with respect to $x, v$, and $l$ :

$$
\begin{array}{r}
F(t, x, v, l)=A(t) x+\frac{B(t)}{\gamma} v+C(t) l+\frac{D(t)}{\gamma}, \\
A(T)=1, B(T)=C(T)=D(T)=0, \\
G(t, x, v, l)=\bar{A}(t) x+\frac{\bar{B}(t)}{\gamma} v+\bar{C}(t) l+\frac{\bar{D}(t)}{\gamma}, \\
\bar{A}(T)=1, \bar{B}(T)=\bar{C}(T)=\bar{D}(T)=0 .
\end{array}
$$

Then

$$
\begin{aligned}
& \pi^{*}(t) \\
& \quad \frac{\left[A(t)\left(\theta-\rho_{p}\right)^{\prime}-\bar{A}(t) \bar{B}(t) \sigma_{v} \sqrt{v} \beta_{v}^{\prime}-\bar{A}(t) \bar{C}(t) \gamma l \beta_{l}^{\prime}\right] \Sigma^{-1}}{\gamma \bar{A}^{-2}(t) x} \\
& +\rho_{p}^{\prime} \Sigma^{-1} .
\end{aligned}
$$

Inserting $\pi^{*}(t)$ into $\mathscr{A}^{\pi^{*}} F(t, x, v, l)-\xi^{\pi^{*}}(t, x, v, l)=0$, we get the following equation:

$$
\begin{aligned}
A_{t} x & +\frac{B_{t}}{\gamma} v+C_{t} l+\frac{D_{t}}{\gamma}+A(t)[k l \\
& \left.+x\left(R(t)-\mu_{p}(t)+\lambda_{p}(t) \sigma_{p}\right)\right]+\frac{B(t)}{\gamma} \alpha(\delta-v) \\
& +C(t) l \alpha_{l}(t)-\frac{\bar{B}^{2}(t)}{2 \gamma} \sigma_{v}^{2} v \\
& +\frac{1}{2 \gamma \bar{A}^{2}(t)}\left[A^{2}(t)\left(\left(\lambda_{p}(t)-\sigma_{p}\right)^{2}+\lambda_{s}^{2} v\right)\right. \\
& -2 A(t) \bar{A}(t) \bar{B}(t) \lambda_{s} \rho_{s v} \sigma_{v} v \\
& -2 A(t) \bar{A}(t) \bar{C}(t) \gamma\left(\lambda_{p}(t)-\sigma_{p}\right)\left(\sigma_{l}-\sigma_{p}\right) l \\
& \left.+\bar{A}^{2}(t) \bar{B}^{2}(t) \sigma_{v}^{2} \rho_{s v}^{2} v\right]=0 .
\end{aligned}
$$

Separating with $x, v, l$, and other terms, we obtain the following ordinary differential equations (ODEs):

$$
\begin{aligned}
A_{t} & +A(t)\left[R(t)-\mu_{p}(t)+\lambda_{p}(t) \sigma_{p}\right]=0 ; \\
B_{t} & -B(t) \alpha-\frac{\bar{B}^{2}(t)}{2} \sigma_{v}^{2}+\frac{1}{2 \bar{A}^{2}(t)}\left[A^{2}(t) \lambda_{s}^{2}\right. \\
& \left.-2 A(t) \bar{A}(t) \bar{B}(t) \lambda_{s} \sigma_{v} \rho_{s v}+\bar{A}^{2}(t) \bar{B}^{2}(t) \sigma_{v}^{2} \rho_{s v}^{2}\right] \\
& =0 ;
\end{aligned}
$$

$$
\begin{aligned}
& C_{t}+k A(t)+C(t) \alpha_{l}(t)-\frac{1}{\bar{A}(t)} A(t) \bar{C}(t)\left(\lambda_{p}(t)\right. \\
& \left.\quad-\sigma_{p}\right)\left(\sigma_{l}-\sigma_{p}\right)=0 ; \\
& D_{t}+\alpha \delta B(t)+\frac{1}{2 \bar{A}^{2}(t)} A^{2}(t)\left(\lambda_{p}(t)-\sigma_{p}\right)^{2}=0 .
\end{aligned}
$$

Now we insert $\pi^{*}(t)$ into $\mathscr{A}^{\pi^{*}} G(t, x, v, l)=0$ and obtain the following equation:

$$
\begin{aligned}
\bar{A}_{t} x & +\frac{\bar{B}_{t}}{\gamma} v+\bar{C}_{t} l+\frac{\bar{D}_{t}}{\gamma}+\bar{A}(t)[k l \\
& \left.+x\left(R(t)-\mu_{p}(t)+\lambda_{p}(t) \sigma_{p}\right)\right]+\frac{\bar{B}(t)}{\gamma} \alpha(\delta-v) \\
& +\bar{C}(t) l \alpha_{l}(t) \\
& +\frac{1}{\gamma \bar{A}(t)}\left[A(t)\left(\left(\lambda_{p}(t)-\sigma_{p}\right)^{2}+\lambda_{s}^{2} v\right)\right. \\
& -\bar{A}(t) \bar{B}(t) \lambda_{s} \sigma_{v} \rho_{s v} v \\
& \left.-\bar{A}(t) \bar{C}(t) \gamma\left(\lambda_{p}(t)-\sigma_{p}\right)\left(\sigma_{l}-\sigma_{p}\right) l\right]=0 .
\end{aligned}
$$

Similarly, separating with $x, v, l$, and other terms, we obtain the following ODEs:

$$
\begin{aligned}
& \bar{A}_{t}+\bar{A}(t)\left[R(t)-\mu_{p}(t)+\lambda_{p}(t) \sigma_{p}\right]=0 ; \\
& \bar{B}_{t}-\bar{B}(t) \alpha+\frac{1}{\bar{A}(t)}\left[A(t) \lambda_{s}^{2}-\bar{A}(t) \bar{B}(t) \lambda_{s} \sigma_{v} \rho_{s v}\right] \\
& \quad=0 ; \\
& \bar{C}_{t}+k \bar{A}(t)+\bar{C}(t)\left[\alpha_{l}(t)-\left(\lambda_{p}(t)-\sigma_{p}\right)\left(\sigma_{l}-\sigma_{p}\right)\right] \\
& \quad=0 ; \\
& \bar{D}_{t}+\alpha \delta \bar{B}(t)+\frac{1}{\bar{A}(t)} A(t)\left(\lambda_{p}(t)-\sigma_{p}\right)^{2}=0 .
\end{aligned}
$$

By using the corresponding boundary conditions, we get the solutions of the ODEs (29) and (31) as follows:

$$
\begin{aligned}
& A(t)=\bar{A}(t)=e^{\int_{t}^{T}\left[R(s)-\mu_{p}(s)+\lambda_{p}(s) \sigma_{p}\right] d s}=e^{\int_{t}^{T} r(s) d s}, \\
& \bar{B}(t)=\frac{\lambda_{s}^{2}}{\alpha+\lambda_{s} \sigma_{v} \rho_{s v}}\left[1-e^{-\left(\alpha+\lambda_{s} \sigma_{v} \rho_{s v}\right)(T-t)}\right],
\end{aligned}
$$




$$
\begin{aligned}
& B(t)=\frac{1}{2} e^{-\alpha(T-t)} \int_{t}^{T} g_{1}(s) e^{\alpha(T-s)} d s, \\
& \bar{C}(t)=C(t)=k \int_{t}^{T} e^{\int_{t}^{s} m(u) d u} e^{\int_{s}^{T} r(u) d u} d s, \\
& D(t)=\alpha \delta \int_{t}^{T} B(s) d s+\frac{1}{2} \int_{t}^{T}\left(\lambda_{p}(s)-\sigma_{p}\right)^{2} d s, \\
& \bar{D}(t)=\alpha \delta \int_{t}^{T} \bar{B}(s) d s+\int_{t}^{T}\left(\lambda_{p}(s)-\sigma_{p}\right)^{2} d s,
\end{aligned}
$$

where $g_{1}(s)=\lambda_{s}^{2}-2 \lambda_{s} \sigma_{v} \rho_{s v} \bar{B}(s)+\bar{B}^{2}(s) \sigma_{v}^{2}\left(\rho_{s v}^{2}-1\right)$ and $m(u)=$ $\mu_{l}-\mu_{p}(u)+\lambda_{p}(u)\left(\sigma_{p}-\sigma_{l}\right)$. Substituting the above results into (27) and after some rearrangement, we can conclude the following theorem.

Theorem 8. For problem $\left(\mathscr{P}_{1}\right)$, the equilibrium investment strategy is

$$
\begin{aligned}
& \pi_{I}^{*}(t)= \begin{cases}{\left[\frac{\lambda_{p}(t)-\sigma_{p}-\lambda_{s} \sigma_{s}+\sigma_{s} \sigma_{v} \rho_{s v} \bar{B}(t)}{\gamma}-l\left(\sigma_{l}-\sigma_{p}\right) \bar{C}(t)\right] \frac{e^{-\int_{t}^{T} r(s) d s}}{x \sigma_{p}}+1,} & \alpha \neq-\lambda_{s} \sigma_{v} \rho_{s v}, \\
{\left[\frac{\lambda_{p}(t)-\sigma_{p}-\lambda_{s} \sigma_{s}+\sigma_{s} \sigma_{v} \rho_{s v} \lambda_{s}^{2}(T-t)}{\gamma}-l\left(\sigma_{l}-\sigma_{p}\right) \bar{C}(t)\right] \frac{e^{-\int_{t}^{T} r(s) d s}}{x \sigma_{p}}+1,} & \alpha=-\lambda_{s} \sigma_{v} \rho_{s v},\end{cases} \\
& \pi_{S}^{*}(t)= \begin{cases}\frac{\lambda_{s} \alpha+\lambda_{s}^{2} \sigma_{v} \rho_{s v} e^{-\left(\alpha+\lambda_{s} \sigma_{v} \rho_{s v}\right)(T-t)}}{\gamma x\left(\alpha+\lambda_{s} \sigma_{v} \rho_{s v}\right)} e^{-\int_{t}^{T} r(s) d s}, & \alpha \neq-\lambda_{s} \sigma_{v} \rho_{s v}, \\
\frac{\lambda_{s}\left[1-\lambda_{s} \sigma_{v} \rho_{s v}(T-t)\right]}{\gamma x} e^{-\int_{t}^{T} r(s) d s}, & \alpha=-\lambda_{s} \sigma_{v} \rho_{s v},\end{cases}
\end{aligned}
$$

and the corresponding equilibrium value function is

$$
V(t, x, v, l)=x e^{\int_{t}^{T} r(s) d s}+\frac{B(t)}{\gamma} v+C(t) l+\frac{D(t)}{\gamma},
$$

where $B(t), C(t)$, and $D(t)$ are given by (34), (35), and (36).

Note that

$$
\begin{aligned}
& \mathbb{E}_{t, x, v, l}\left[X^{\pi^{*}}(T)\right] \\
& =x e^{\int_{t}^{T} r(s) d s}+\frac{1}{\gamma}[\bar{B}(t) v+\bar{D}(t)]+\bar{C}(t) l, \\
& \operatorname{Var}_{t, x, v, l}\left[X^{\pi^{*}}(T)\right]=H(t, x, v, l)-G^{2}(t, x, v, l) \\
& =\frac{2}{\gamma}[G(t, x, v, l)-F(t, x, v, l)] \\
& =\frac{2}{\gamma^{2}}[(\bar{B}(t)-B(t)) v+\bar{D}(t)-D(t)] .
\end{aligned}
$$

Thus we obtain the following so-called equilibrium efficient frontier, which reflects the relationship between the investment risk and return under the equilibrium strategy.

Corollary 9. For $M V$ problem $\left(\mathscr{P}_{1}\right)$, based on state $(t, x, v, l)$, its equilibrium efficient frontier is

$$
\begin{aligned}
\mathbb{E}_{t, x, v, l}\left[X^{\pi^{*}}(T)\right]= & x e^{\int_{t}^{T} r(s) d s}+\bar{C}(t) l \\
& +\bar{N}(t, v, l) \sqrt{\operatorname{Var}_{t, x, v, l}\left[X^{\pi^{*}}(T)\right]},
\end{aligned}
$$

$$
\begin{aligned}
& \text { where } \bar{N}(t, v, l)= \\
& \sqrt{2[(\bar{B}(t)-B(t)) v+\bar{D}(t)-D(t)]}
\end{aligned} . \quad[\bar{B}(t) v+\bar{D}(t)] /
$$

Remark 10. By (38) and (39), we find that the equilibrium investment proportion in the stock has the similar expression as in [24], although their study focuses on the optimal investment and reinsurance problem. In addition, the equilibrium investment money in the stock is only the function of time $t$, which is independent of the current wealth. However, for the inflation-linked bond, the equilibrium investment money depends on current wealth, and the more the wealth is, the more the inflation-linked bond is invested. This result is consistent with the economic intuition, considering that when the manager has more wealth, the sensitivity to the inflation risk becomes higher, and the corresponding hedging demand is improved.

Remark 11. In view of (38), we find that if $\sigma_{p}>\sigma_{l}$, that is, the volatility rate of the inflation is larger than that of the salary income, the equilibrium investment amount in the inflationlinked bond has positive relationship with the salary level. Otherwise, it has negative relationship with the salary level.

Remark 12. From (42) and (43), if $\operatorname{Var}_{t, x, v, l}\left[X^{\pi}(T)\right]=0$, it means that the pension fund manager bears no risk at all under the state $(t, x, v, l)$; this is equivalent to the case of $\gamma \rightarrow \infty$; that is, the pension fund manager is fully averse to risk. In this case, $\pi_{S}^{*}(t)=0$ and $\pi_{I}^{*}(t)=1-\left(\left(\sigma_{l}-\right.\right.$ $\left.\left.\sigma_{p}\right) / x \sigma_{p}\right) l \bar{C}(t) e^{-\int_{t}^{T} r(s) d s}$. This indicates that the manager will only invest all the fund in the inflation-linked bond and the risk-free money market account and obtain the expected real terminal wealth of $x e^{\int_{t}^{T} r(s) d s}+\bar{C}(t) l$ at retirement time $T$. It consists of two parts. The first part is the accumulation of the initial wealth $x$, and the second part can be seen as the accumulation of the contribution. 
Remark 13. If we do not consider the risk of stochastic volatility of the stock price; that is, let $\alpha=0, \sigma_{v}=0$; then the equilibrium strategy is simplified as

$$
\begin{aligned}
& \pi_{I}^{*}(t) \\
& =\left[\frac{\lambda_{p}(t)-\sigma_{p}-\lambda_{s} \sigma_{s}}{\gamma}-l\left(\sigma_{l}-\sigma_{p}\right) \bar{C}(t)\right] \frac{e^{-\int_{t}^{T} r(s) d s}}{x \sigma_{p}} \\
& \quad+1, \\
& \pi_{S}^{*}(t)=\frac{\lambda_{s} e^{-\int_{t}^{T} r(s) d s}}{\gamma x} .
\end{aligned}
$$

In this case, we find that $\pi_{S}^{*}(t)$ is consistent with the result of the ordinary MV portfolio problem under the BlackScholes market (see [11]). This indicates that the contribution of the salary income has no influence on the equilibrium investment amount of the stock. Furthermore, if we do not consider the risk of inflation, that is, $\mu_{p}(t)=\sigma_{p}=\sigma_{s}=0$, then $R(t)=r(t)$ and the inflation-linked bond is degenerated to the risk-free money market account.

\section{Problem Formulation after Retirement and Verification Theorem}

After retirement, we assume that the pension plan member purchases a paid-up annuity during the time horizon $[T, T+$ $N]$. Thus the objective function of the pension plan manager is to maximize the expected surplus after paying off the benefit to the member and minimize the variance of the surplus at time $T_{1}=T+N$. So, at the state $(t, x, v) \in \mathscr{G}_{1}$, the manager faces the following optimal control problem:

$$
\begin{gathered}
\widehat{V}(t, x, v)=\sup _{\widehat{\pi} \in \widehat{\Pi}(t, x, v)}\left\{\mathbb{E}_{t, x, v}\left[X^{\widehat{\pi}}\left(T_{1}\right)\right]\right. \\
\left.-\frac{\gamma}{2} \operatorname{Var}_{t, x, v}\left[X^{\widehat{\pi}}\left(T_{1}\right)\right]\right\} .
\end{gathered}
$$

Similarly to the method of Section 3 , we denote $y^{\widehat{\pi}}(t, x, v)=$ $\mathbb{E}_{t, x, v}\left[X^{\hat{\pi}}\left(T_{1}\right)\right], z^{\hat{\pi}}(t, x, v)=\mathbb{E}_{t, x, v}\left[\left(X^{\hat{\pi}}\left(T_{1}\right)\right)^{2}\right]$. Then we can rewrite

$$
\begin{aligned}
\widehat{V} & (t, x, v) \\
& =\sup _{\widehat{\pi} \in \Pi(t, x, v)}\left\{\widehat{f}\left(t, x, v, y^{\widehat{\pi}}(t, x, v), z^{\widehat{\pi}}(t, x, v)\right)\right\},
\end{aligned}
$$

where $\widehat{f}(t, x, v, y, z)=y-(\gamma / 2)\left(z-y^{2}\right)$.

4.1. Verification Theorem. In this subsection, we first give the definition of equilibrium strategy after retirement and then give the verification theorem which is satisfied by the equilibrium value function. Now, for any $\phi(t, x, v) \in C^{1,2,2}\left(\mathscr{G}_{1}\right)$, let set $\widehat{D}_{p}\left(\mathscr{G}_{1}\right)=\left\{\phi(t, x, v) \mid \phi(t, x, v) \in C^{1,2,2}\left(\mathscr{G}_{1}\right)\right.$ and all firstorder partial derivatives of $\phi(\cdot, x, v)$ satisfy the polynomial growth condition on $\left.\mathcal{O}_{1}\right\}$, and denote differential operator as

$$
\begin{aligned}
& \mathscr{L}^{\widehat{\pi}} \phi(t, x, v) \\
& =\phi_{t}+\phi_{x}\left[x\left(R-\mu_{p}+\sigma_{p}^{2}+\widehat{\pi} \Sigma\left(\theta-\rho_{p}\right)\right)-q\right] \\
& \quad+\phi_{v} \alpha(\delta-v)+\frac{1}{2} \phi_{x x} x^{2}\left(\hat{\pi} \Sigma-\rho_{p}^{\prime}\right)\left(\hat{\pi} \Sigma-\rho_{p}^{\prime}\right)^{\prime} \\
& \quad+\frac{1}{2} \phi_{v v} \sigma_{v}^{2} v+\phi_{x v} x \sigma_{v} \sqrt{v}\left(\hat{\pi} \Sigma-\rho_{p}^{\prime}\right) \beta_{v} .
\end{aligned}
$$

Definition 14. For any given initial state $(t, x, v) \in \mathscr{G}_{1}$, consider an admissible strategy $\hat{\pi}^{*}(t, x, v)$ and choose three real numbers $\tau>0, \bar{\pi}_{I}$, and $\bar{\pi}_{B}$; one defines the following strategy:

$$
\begin{aligned}
& \pi_{\tau}(s, \bar{x}, \bar{v}) \\
& \quad= \begin{cases}\left(\bar{\pi}_{I}, \bar{\pi}_{B}\right), & t \leqslant s<t+\tau,(\bar{x}, \bar{v}) \in \mathcal{O}_{1} ; \\
\hat{\pi}^{*}(s, \bar{x}, \bar{v}), & t+\tau \leqslant s<T_{1},(\bar{x}, \bar{v}) \in \mathcal{O}_{1} .\end{cases}
\end{aligned}
$$

If $\lim _{\tau \rightarrow 0} \inf \left(\left(\widehat{f}^{\hat{\pi}^{*}}(t)-\widehat{f}^{\pi_{\tau}}(t)\right) / \tau\right) \geqslant 0 \forall\left(\bar{\pi}_{I}, \bar{\pi}_{B}\right) \in \mathbb{R} \times \mathbb{R}$, then $\widehat{\pi}^{*}(t, x, v)$ is called an equilibrium strategy, and the equilibrium value function is defined as $\widehat{V}(t, x, v)=\widehat{f}(t, x, v$, $\left.y^{\hat{\pi}^{*}}(t, x, v), z^{\hat{\pi}^{*}}(t, x, v)\right)$, where $\hat{f}^{\hat{\pi}}(t)=\hat{f}\left(t, x, v, y^{\widehat{\pi}}(t, x, v)\right.$, $\left.z^{\hat{\pi}}(t, x, v)\right)$ for short.

Theorem 15 (verification theorem). For the optimal asset allocation problem $\left(\mathscr{P}_{2}\right)$, if there exist three real value functions $\widehat{F}(t, x, v), \widehat{G}(t, x, v)$, and $\widehat{H}(t, x, v) \in \widehat{D}_{p}\left(\mathscr{G}_{1}\right)$ satisfying the following conditions: $\forall(t, x, v) \in \mathscr{G}_{1}$,

$$
\begin{aligned}
& \sup _{\widehat{\pi} \in \widehat{\Pi}(t, x, v)}\left\{\mathscr{L}^{\widehat{\pi}} \widehat{F}(t, x, v)-\varphi^{\widehat{\pi}}(t, x, v)\right\}=0, \\
& \widehat{F}\left(T_{1}, x, v\right)=\widehat{f}\left(T_{1}, x, v, x, x^{2}\right), \\
& \mathscr{L}^{\widehat{\pi}^{*}} \widehat{G}(t, x, v)=0 \\
& \widehat{G}\left(T_{1}, x, v\right)=x \\
& \mathscr{L}^{\widehat{\pi}^{*}} \widehat{H}(t, x, v)=0 \\
& \widehat{H}\left(T_{1}, x, v\right)=x^{2}
\end{aligned}
$$

where $\widehat{\pi}^{*}=\arg \sup _{\widehat{\pi} \in \widehat{\Pi}(t, x, v)}\left\{\mathscr{L}^{\widehat{\pi}} \widehat{F}(t, x, v)-\varphi^{\widehat{\pi}}(t, x, v)\right\}$, then $\widehat{V}(t, x, v)=\widehat{F}(t, x, v), y^{\widehat{\pi}^{*}}(t, x, v)=\widehat{G}(t, x, v)$, and $z^{\widehat{\pi}^{*}}(t, x$, $v)=\widehat{H}(t, x, v)$, and $\widehat{\pi}^{*}$ is the equilibrium strategy, where

$$
\varphi^{\hat{\pi}}(t, x, v)
$$

$$
\begin{aligned}
= & f_{t}+\left[x\left(R-\mu_{p}+\sigma_{p}^{2}+\widehat{\pi} \Sigma\left(\theta-\rho_{p}\right)\right)-q\right] f_{x} \\
& +f_{v} \alpha(\delta-v)+\frac{1}{2}\left(\widehat{\pi} \Sigma-\rho_{p}^{\prime}\right)\left(\widehat{\pi} \Sigma-\rho_{p}^{\prime}\right)^{\prime} x^{2} \widehat{U}_{1}^{\widehat{\pi}} \\
& +\frac{1}{2} \sigma_{v}^{2} v \widehat{U}_{2}^{\widehat{\pi}}+\sigma_{v} \sqrt{v} x\left(\widehat{\pi}^{\prime} \Sigma-\rho_{p}^{\prime}\right) \beta_{v} \widehat{U}_{3}^{\widehat{\pi}},
\end{aligned}
$$




$$
\begin{aligned}
\widehat{U}_{1}^{\hat{\pi}} & \\
= & f_{x x}+f_{y y}\left(y_{x}^{\hat{\pi}}\right)^{2}+f_{z z}\left(z_{x}^{\widehat{\pi}}\right)^{2}+2 f_{x y} y_{x}^{\hat{\pi}}+2 f_{x z} z_{x}^{\widehat{\pi}} \\
& +2 f_{y z} y_{x}^{\hat{\pi}} z_{x}^{\hat{\pi}},
\end{aligned}
$$

and $f=f(t, x, v, y, z), y^{\hat{\pi}}=y^{\hat{\pi}}(t, x, v)$, and $z^{\hat{\pi}}=z^{\hat{\pi}}(t, x, v)$.

The proof is similar to Theorem 7 and here we omit it.

4.2. Solution to the Equilibrium Strategy. Using Theorem 15, now we calculate the equilibrium strategy and corresponding equilibrium value function. By the similar method to that in Section 3, we can obtain the equilibrium strategy as follows: $\hat{\pi}^{*}(t)$

$$
\begin{aligned}
= & -\frac{\left[\widehat{F}_{x}\left(\theta-\rho_{p}\right)^{\prime}+\left(\widehat{F}_{x v}-\gamma \widehat{G}_{x} \widehat{G}_{v}\right) \sigma_{v} \sqrt{v} \beta_{v}^{\prime}\right] \Sigma^{-1}}{\left(\widehat{F}_{x x}-\gamma \widehat{G}_{x}^{2}\right) x} \\
& +\rho_{p}^{\prime} \Sigma^{-1} .
\end{aligned}
$$

We suppose $\widehat{F}(t, x, v)$ and $\widehat{G}(t, x, v)$ have the following linear forms with respect to $x$ and $v$ :

$$
\begin{aligned}
& \widehat{F}(t, x, v)=a(t) x+\frac{b(t)}{\gamma} v+\frac{c(t)}{\gamma}, \\
& a\left(T_{1}\right)=1, b\left(T_{1}\right)=c\left(T_{1}\right)=0, \\
& \widehat{G}(t, x, v)=\bar{a}(t) x+\frac{\bar{b}(t)}{\gamma} v+\frac{\bar{c}(t)}{\gamma}, \\
& \bar{a}\left(T_{1}\right)=1, \bar{b}\left(T_{1}\right)=\bar{c}\left(T_{1}\right)=0 .
\end{aligned}
$$

Then

$$
\begin{aligned}
\widehat{\pi}^{*}(t)= & \frac{\left[a(t)\left(\theta-\rho_{p}\right)^{\prime}-\bar{a}(t) \bar{b}(t) \sigma_{v} \sqrt{v} \beta_{v}^{\prime}\right] \Sigma^{-1}}{\gamma \bar{a}^{2}(t) x} \\
& +\rho_{p}^{\prime} \Sigma^{-1} .
\end{aligned}
$$

Inserting (52) into $\mathscr{L}^{\widehat{\pi}^{*}} \widehat{F}(t, x, v)-\varphi^{\hat{\pi}^{*}}(t, x, v)=0$, we get the following equation:

$$
\begin{aligned}
a_{t} x & +\frac{b_{t}}{\gamma} v+\frac{c_{t}}{\gamma}+a(t)\left[x\left(R-\mu_{p}+\lambda_{p}(t) \sigma_{p}\right)-q\right] \\
& +\frac{b(t)}{\gamma} \alpha(\delta-v)-\frac{\bar{b}^{2}(t)}{2 \gamma} \sigma_{v}^{2} v \\
& +\frac{1}{2 \gamma \bar{a}^{2}(t)}\left[a^{2}(t)\left(\left(\lambda_{p}(t)-\sigma_{p}\right)^{2}+\lambda_{s}^{2} v\right)\right.
\end{aligned}
$$

$$
\begin{aligned}
& \left.-2 a(t) \bar{a}(t) \bar{b}(t) \lambda_{s} \rho_{s v} \sigma_{v} v+\bar{a}^{2}(t) \bar{b}^{2}(t) \sigma_{v}^{2} \rho_{s v}^{2} v\right] \\
& =0
\end{aligned}
$$

Separating with $x, v$, and other terms, we obtain the following ODEs:

$$
\begin{aligned}
& a_{t}+a(t)\left[R(t)-\mu_{p}(t)+\lambda_{p}(t) \sigma_{p}\right]=0 ; \\
& b_{t}-b(t) \alpha-\frac{\bar{b}^{2}(t)}{2} \sigma_{v}^{2}+\frac{1}{2 \bar{a}^{2}(t)}\left[a^{2}(t) \lambda_{s}^{2}\right. \\
& \left.\quad-2 a(t) \bar{a}(t) \bar{b}(t) \lambda_{s} \sigma_{v} \rho_{s v}+\bar{a}^{2}(t) \bar{b}^{2}(t) \sigma_{v}^{2} \rho_{s v}^{2}\right]=0 ; \\
& c_{t}+\alpha \delta b(t)+\frac{1}{2 \bar{a}^{2}(t)} a^{2}(t)\left(\lambda_{p}(t)-\sigma_{p}\right)^{2}-\gamma a(t) q \\
& \quad=0 .
\end{aligned}
$$

Now we insert $\widehat{\pi}^{*}(t)$ into $\mathscr{L}^{\widehat{\pi}^{*}} \widehat{G}(t, x, v)=0$ and obtain the following equation:

$$
\begin{aligned}
\bar{a}_{t} x+\frac{\bar{b}_{t}}{\gamma} v+\frac{\bar{c}_{t}}{\gamma}+\bar{a}(t)\left[x\left(R(t)-\mu_{p}(t)+\lambda_{p}(t) \sigma_{p}\right)\right. \\
-q]+\frac{\bar{b}(t)}{\gamma} \alpha(\delta-v) \\
+\frac{1}{\gamma \bar{a}(t)}\left[a(t)\left(\left(\lambda_{p}(t)-\sigma_{p}\right)^{2}+\lambda_{s}^{2} v\right)\right. \\
\left.-\bar{a}(t) \bar{b}(t) \lambda_{s} \sigma_{v} \rho_{s v} v\right]=0 .
\end{aligned}
$$

Similarly, separating with $x, v$, and other terms, we have the following ODEs:

$$
\begin{aligned}
\bar{a}_{t}+\bar{a}(t)\left[R(t)-\mu_{p}(t)+\lambda_{p}(t) \sigma_{p}\right] & =0 ; \\
\bar{b}_{t}-\bar{b}(t) \alpha+\frac{1}{\bar{a}(t)}\left[a(t) \lambda_{s}^{2}-\bar{a}(t) \bar{b}(t) \lambda_{s} \sigma_{v} \rho_{s v}\right] & =0 ; \\
\bar{c}_{t}+\alpha \delta \bar{b}(t)+\frac{1}{\bar{a}(t)} a(t)\left(\lambda_{p}(t)-\sigma_{p}\right)^{2}-\gamma \bar{a}(t) q & =0 .
\end{aligned}
$$

By using the boundary conditions, we solve the above ODEs and yield

$$
\begin{aligned}
a(t)= & \bar{a}(t)=e^{\int_{t}^{T_{1}} r(s) d s}, \\
\bar{b}(t)= & \frac{\lambda_{s}^{2}}{\alpha+\lambda_{s} \sigma_{v} \rho_{s v}}\left[1-e^{-\left(\alpha+\lambda_{s} \sigma_{v} \rho_{s v}\right)\left(T_{1}-t\right)}\right], \\
b(t)= & \frac{1}{2} e^{-\alpha\left(T_{1}-t\right)} \int_{t}^{T_{1}} g_{2}(s) e^{\alpha\left(T_{1}-s\right)} d s, \\
c(t)= & \alpha \delta \int_{t}^{T_{1}} b(s) d s+\frac{1}{2} \int_{t}^{T_{1}}\left(\lambda_{p}(s)-\sigma_{p}\right)^{2} d s \\
& -\gamma q \int_{t}^{T_{1}} e^{\int_{s}^{T_{1}} r(u) d u} d s, \\
\bar{c}(t)= & \alpha \delta \int_{t}^{T_{1}} \bar{b}(s) d s+\int_{t}^{T_{1}}\left(\lambda_{p}(s)-\sigma_{p}\right)^{2} d s \\
& -\gamma q \int_{t}^{T_{1}} e^{\int_{s}^{T_{1}} r(u) d u} d s,
\end{aligned}
$$


where $g_{2}(s)=\lambda_{s}^{2}-2 \lambda_{s} \sigma_{v} \rho_{s v} \bar{b}(s)+\bar{b}^{2}(s) \sigma_{v}^{2}\left(\rho_{s v}^{2}-1\right)$. Inserting (57) and (58) into (52), we have the following theorem.
Theorem 16. For problem $\left(\mathscr{P}_{2}\right)$, the equilibrium investment strategy is

$$
\begin{aligned}
& \hat{\pi}_{I}^{*}(t)= \begin{cases}{\left[\lambda_{p}(t)-\sigma_{p}-\frac{\lambda_{s} \sigma_{s}\left[\alpha+\lambda_{s} \sigma_{v} \rho_{s v} e^{-\left(\alpha+\lambda_{s} \sigma_{v} \rho_{s v}\right)\left(T_{1}-t\right)}\right]}{\alpha+\lambda_{s} \sigma_{v} \rho_{s v}}\right] \frac{e^{-\int_{t}^{T_{1}} r(s) d s}}{\gamma x \sigma_{p}}+1,} & \alpha \neq-\lambda_{s} \sigma_{v} \rho_{s v}, \\
\frac{\lambda_{p}(t)-\sigma_{p}-\lambda_{s} \sigma_{s}+\sigma_{s} \sigma_{v} \rho_{s v} \lambda_{s}^{2}\left(T_{1}-t\right)}{\gamma x \sigma_{p}} e^{-\int_{t}^{T_{1}} r(s) d s}+1, & \alpha=-\lambda_{s} \sigma_{v} \rho_{s v},\end{cases} \\
& \hat{\pi}_{S}^{*}(t)= \begin{cases}\frac{\lambda_{s} \alpha+\lambda_{s}^{2} \sigma_{v} \rho_{s v} e^{-\left(\alpha+\lambda_{s} \sigma_{v} \rho_{s v}\right)\left(T_{1}-t\right)}}{\gamma x\left(\alpha+\lambda_{s} \sigma_{v} \rho_{s v}\right)} e^{-\int_{t}^{T_{1}} r(s) d s}, & \alpha \neq-\lambda_{s} \sigma_{v} \rho_{s v}, \\
\frac{\lambda_{s}\left[1-\lambda_{s} \sigma_{v} \rho_{s v}\left(T_{1}-t\right)\right]}{\gamma x} e^{-\int_{t}^{T_{1}} r(s) d s}, & \alpha=-\lambda_{s} \sigma_{v} \rho_{s v},\end{cases}
\end{aligned}
$$

and the corresponding equilibrium value function is

$$
V(t, x, v, l)=x e^{\int_{t}^{T_{1}} r(s) d s}+\frac{b(t)}{\gamma} v+\frac{c(t)}{\gamma}
$$

where $b(t)$ and $c(t)$ are given by (59) and (60).

After some calculations, we have the following corollary.
Corollary 17. Based on state $(t, x, v)$, for the $M V$ problem $\left(\mathscr{P}_{2}\right)$, the equilibrium efficient frontier is

$$
\begin{aligned}
\mathbb{E}_{t, x, v}\left[X^{\hat{\pi}^{*}}\left(T_{1}\right)\right]= & x e^{\int_{t}^{T_{1}} r(s) d s}-q \int_{t}^{T_{1}} e^{\int_{s}^{T_{1}} r(u) d u} d s \\
& +\widehat{N}(t, v) \sqrt{\operatorname{Var}_{t, x, v}\left[X^{\hat{\pi}^{*}}\left(T_{1}\right)\right]},
\end{aligned}
$$

where

$$
\widehat{N}(t, v)=\frac{\bar{b}(t) v+\alpha \delta \int_{t}^{T_{1}}\left[\bar{b}(s)+\left(\lambda_{p}(s)-\sigma_{p}\right)^{2}\right] d s}{\sqrt{2\left[(\bar{b}(t)-b(t)) v+\alpha \delta \int_{t}^{T_{1}}\left(\bar{b}(s)-b(s)+(1 / 2)\left(\lambda_{p}(s)-\sigma_{p}\right)^{2}\right) d s\right]}} .
$$

Remark 18. From (62) and (64), we find that, at the state $(t, x, v)$, if manager does not undertake any risk in the later time, that is, let $\operatorname{Var}_{t, x, v}\left[X^{\pi^{*}}\left(T_{1}\right)\right]=0$ or $\gamma \rightarrow \infty$, he/she will only invest all the surplus into the inflation-linked bond after paying off a continuous annuity with real value of $q$ for each time. Then at terminal time $T_{1}$ the expected real net surplus is $x e^{\int_{t}^{T_{1}} r(s) d s}-q \int_{t}^{T_{1}} e^{\int_{s}^{T_{1}} r(u) d u} d s$. It includes two parts, where the first part is the accumulation of the initial real wealth $x$ and the second part is the accumulated payment of the real benefit until time $T_{1}$.

\section{Sensitivity Analysis}

In this section, we analyze the influence of the different parameters on the equilibrium strategy and the equilibrium efficient frontier. Here we only analyze the case before retirement and suppose that $R(t), r(t)$, and $\mu_{p}(t)$ are all deterministic constants for simplicity, and the basic parameters are set in Table 1.

In addition, the initial values are $\bar{x}_{0}=2, p_{0}=1, v_{0}=0.15$, and $\bar{l}_{0}=0.5$. Note that the parameter $\lambda_{p}=0.015$ based on (7). To obtain the evolution process of the investment strategy over time, using Monte Carlo method, we simulate the trajectory of the optimal wealth process with 300 times and obtain the mean investment proportion of the three assets.

5.1. The Analysis of Equilibrium Strategies. This subsection works on analyzing the influence of the inflation, the salary income, and the risk aversion level of the manager on the equilibrium strategy in the period before retirement. Since the sign of the first-order derivative about the investment strategies to some parameters could not be obtained directly, we only give the intuitive analysis.

Figure 1 depicts the mean equilibrium investment proportion of the three assets under the basic parameter setting. We find that the proportion of the stock decreases gradually from $40 \%$ to almost $20 \%$ during time horizon $[0,10]$, and the proportion of the money market account also decreases with time. However, the proportion of the inflation-linked bond is relatively low at the beginning; even short selling happened at time 0 , but, after about 10 years, the proportion increases to almost $20 \%$. In the following analysis, we use Figure 1 as a benchmark. Figure 2 shows the mean investment proportion when $\gamma=0.8$. Compared with Figure 1 , the whole equilibrium investment proportion of the stock moves down when the risk aversion level of the manager increases from $\gamma=0.6$ to 0.8 (this result also can be found from (39), since 
TABLE 1: The basic parameters value.

\begin{tabular}{ccccccccccccccc}
\hline$R$ & $r$ & $\gamma$ & $\mu_{p}$ & $\sigma_{p}$ & $\lambda_{s}$ & $\alpha$ & $\delta$ & $\sigma_{v}$ & $\sigma_{s}$ & $\rho_{s v}$ & $\mu_{l}$ & $\sigma_{l}$ & $k$ & $T$ \\
\hline 0.05 & 0.02 & 0.6 & 0.033 & 0.2 & 0.6 & 0.5 & 0.2 & 0.1 & 0.5 & -0.3 & 0.05 & 0.1 & 0.2 & 10
\end{tabular}

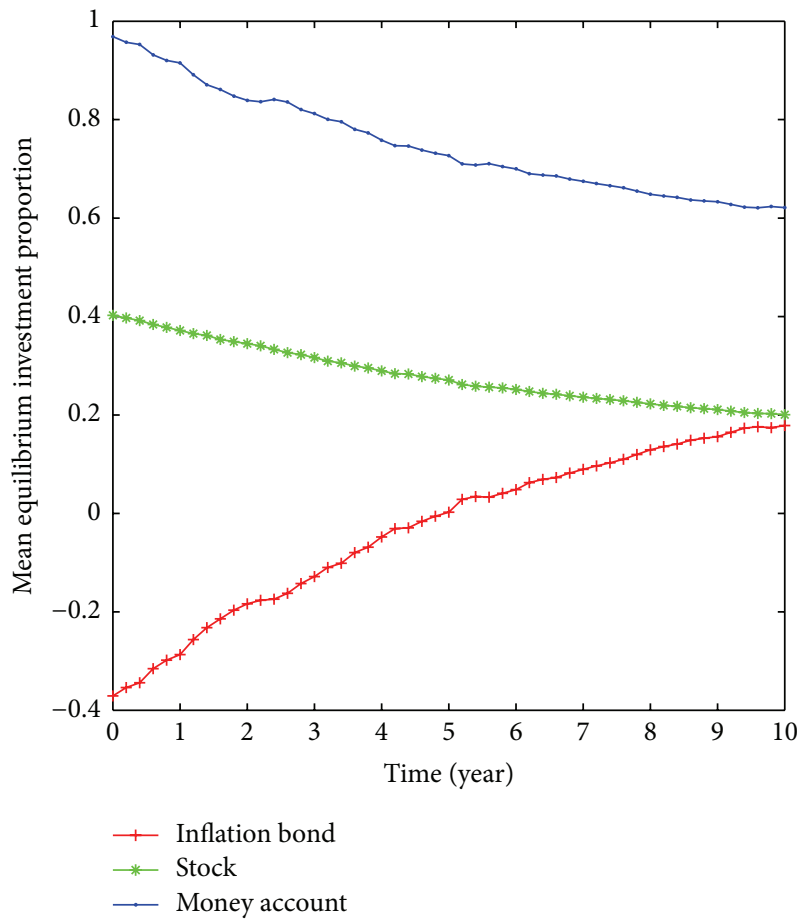

FIGURE 1: The mean investment proportion.

under the basic parameter setting of this section, $\partial \pi_{S}^{*} / \partial \gamma<$ $0)$. On the contrary, the proportion of the inflation-linked bond increases under the higher risk aversion level. The case when the expectation inflation rate $\mu_{p}=0.042$ is illustrated in Figure 3. Since $\mu_{p}$ is higher than that in Figure 1, the manager invests more wealth in the inflation-linked bond to hedge the inflation risk. Similarly, Figure 4 shows that if the expected salary growth rate $\mu_{l}$ increases, the investment proportion in the inflation bond also increases. This means that the more the wealth contributed to the pension fund, the higher the demand to hedge the inflation risk. We note that the proportion of the stock is independent of $\mu_{p}$ and $\mu_{l}$ in (39), so the investment proportion in the stock is the same as in Figure 1.

5.2. The Analysis of the Equilibrium Efficient Frontier. In this subsection, we analyze the influence of different parameters on the equilibrium efficient frontier in the investment period before retirement. Without loss of generality, we only analyze the efficient frontier at time 0 .

Figure 5 shows the efficient frontiers for the different expected inflation rate $\mu_{p}$. Generally speaking, the larger the value of $\mu_{p}$, the lower the real value of the wealth. So, as shown in Figure 5, the expected real terminal wealth has a negative relationship with the expected inflation rate when the variance of the real terminal wealth is fixed. Figure 6 reveals the relationship between the efficient frontier

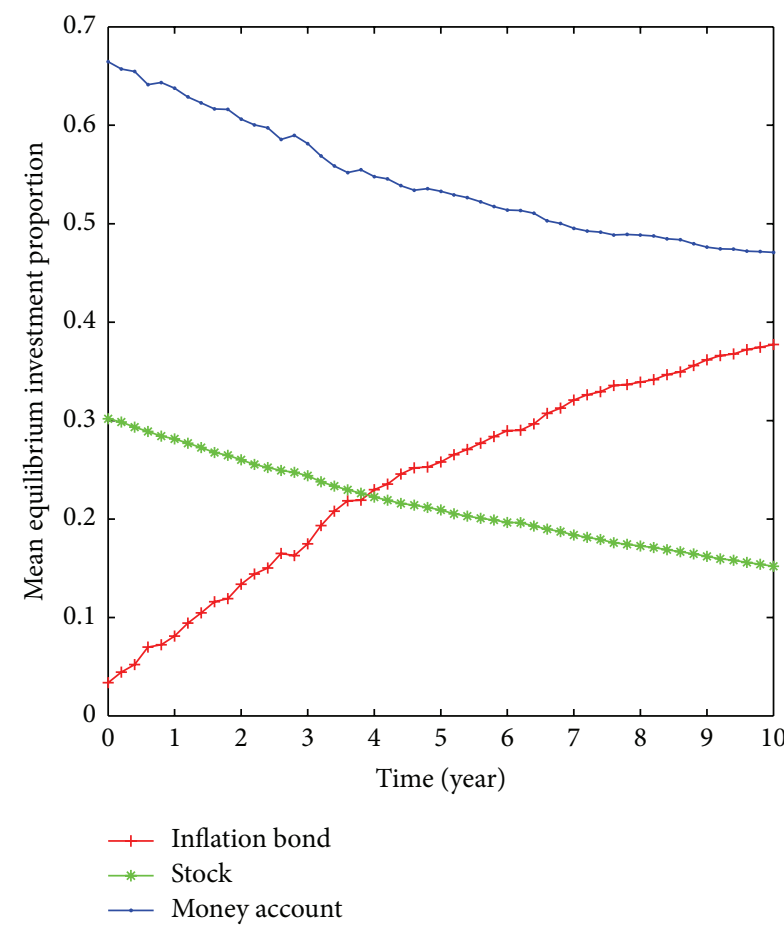

FIGURE 2: $\gamma=0.8$.

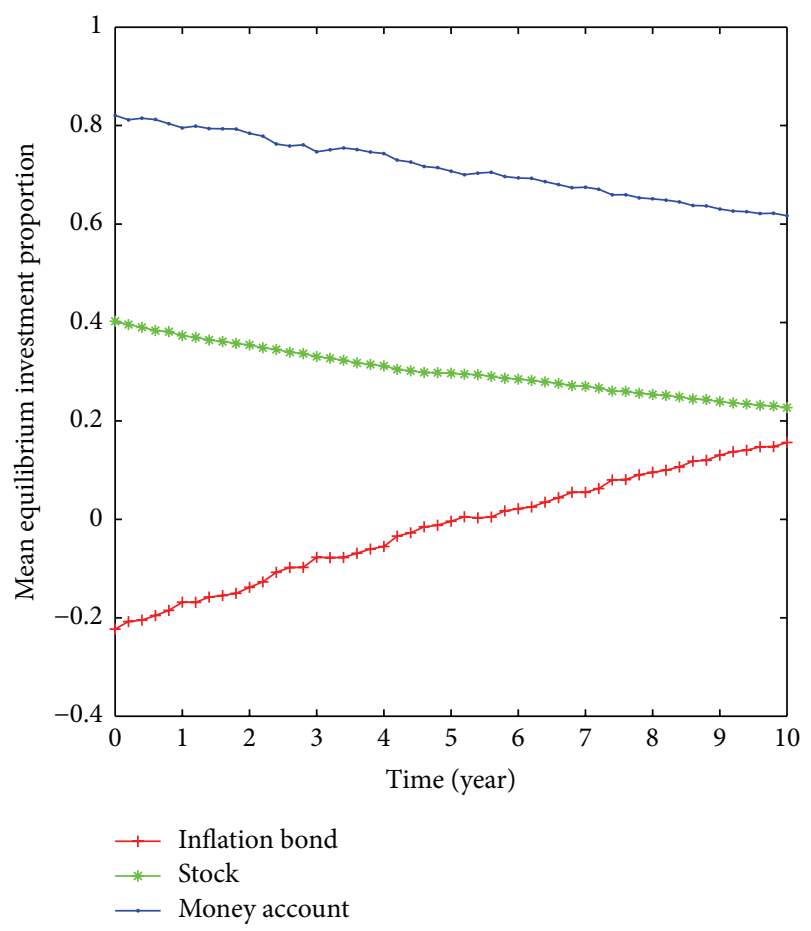

FIGURE 3: $\mu_{p}=0.042$.

and the volatility of the inflation rate $\sigma_{p}$. We find that the higher $\sigma_{p}$ leads to the efficient frontier moving upwards. The influence of the $\delta$ on efficient frontier is illustrated in Figure 7, which shows that the value of the expected volatility of the stock has positive relationship with the efficient frontier. Figure 8 depicts the influence of $\rho_{s v}$ on the efficient frontier. 


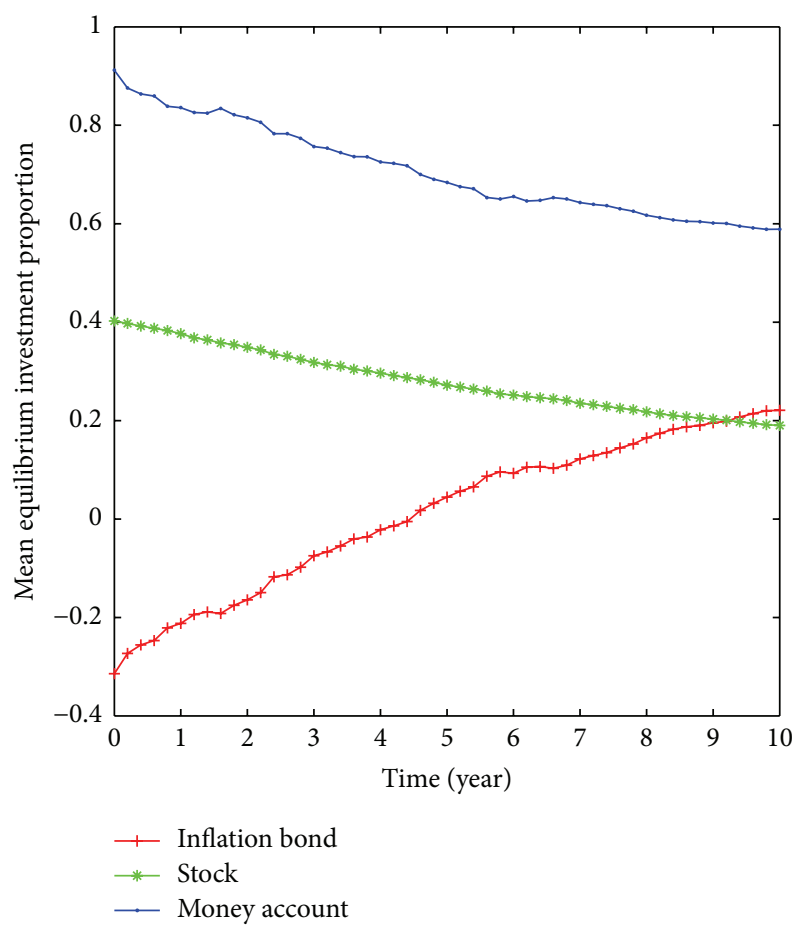

FIGURE 4: $\mu_{l}=0.09$.

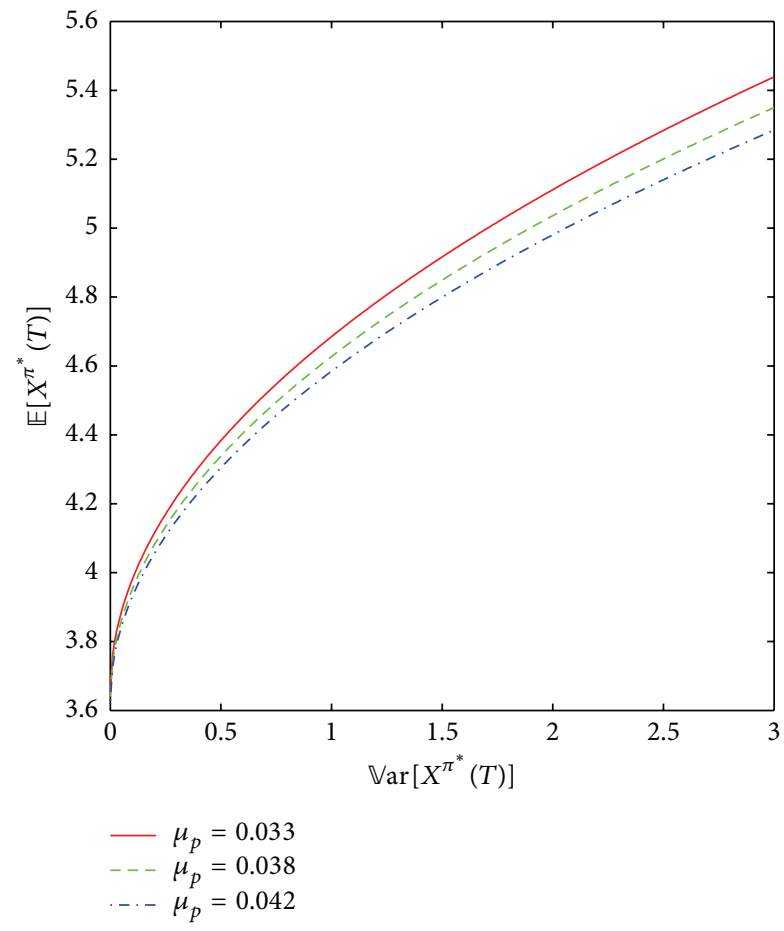

FIgURE 5: The effect of $\mu_{p}$ on efficient frontier.

This indicates that the stronger negative relationship between the stock price $S(t)$ and the volatility $V(t)$ will lead to the efficient frontier moving upwards. Figure 9 shows that the expected growth rate of the salary has a positive influence on the efficient frontier; that is, with increasing of $\mu_{l}$, the efficient frontier moves upwards. In addition, the intersection

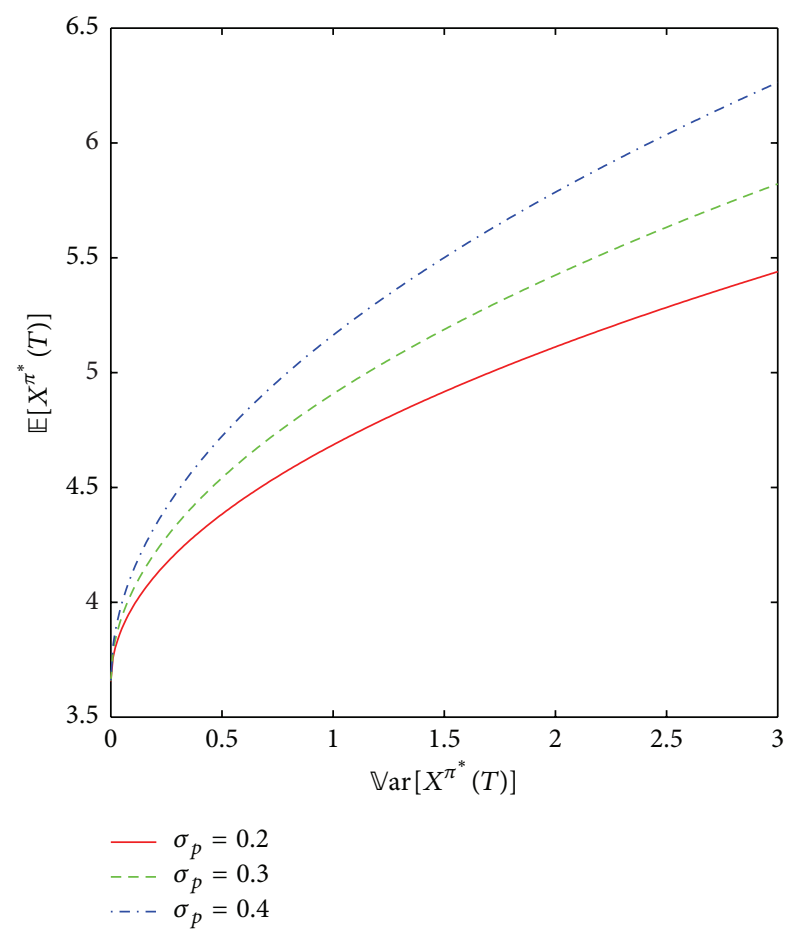

Figure 6: The effect of $\sigma_{p}$ on efficient frontier.

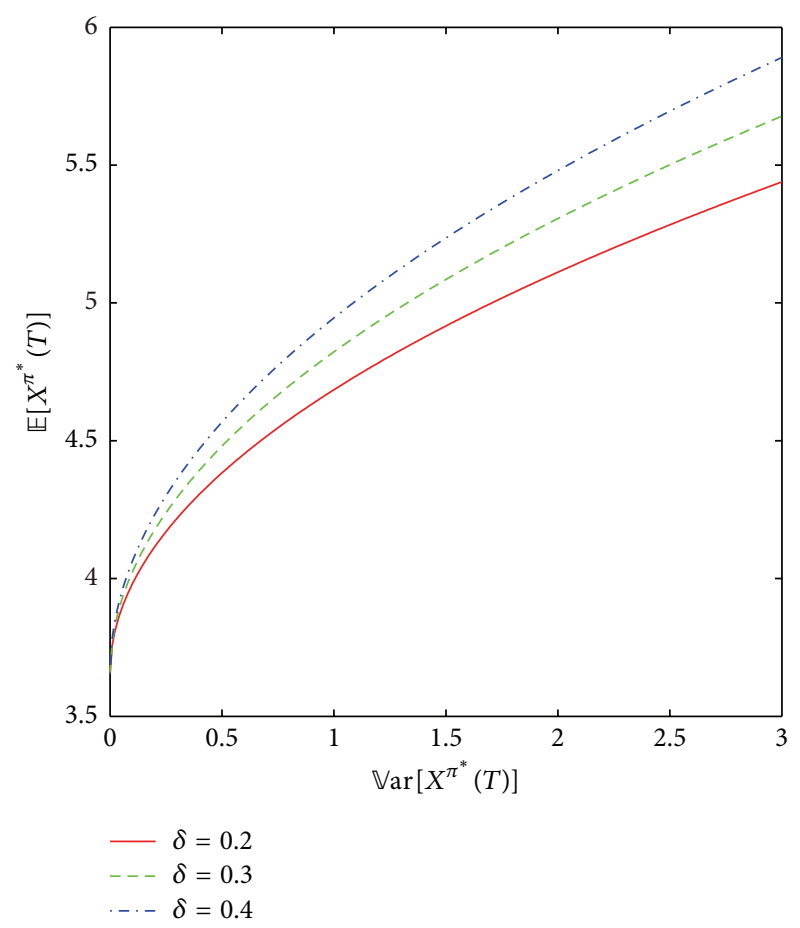

FIgURE 7: The effect of $\delta$ on efficient frontier.

of the efficient frontier and the $\mathbb{E}\left[X^{\pi^{*}}(T)\right]$ axis is bigger if $\mu_{l}$ is larger. This result also can be obtained from Remark 12. In Figure 10, the influence of the salary volatility $\sigma_{l}$ on efficient frontier is revealed. We find that the larger the $\sigma_{l}$, the more the uncertainty about the salary income, which leads to the efficient frontier moving downwards. 


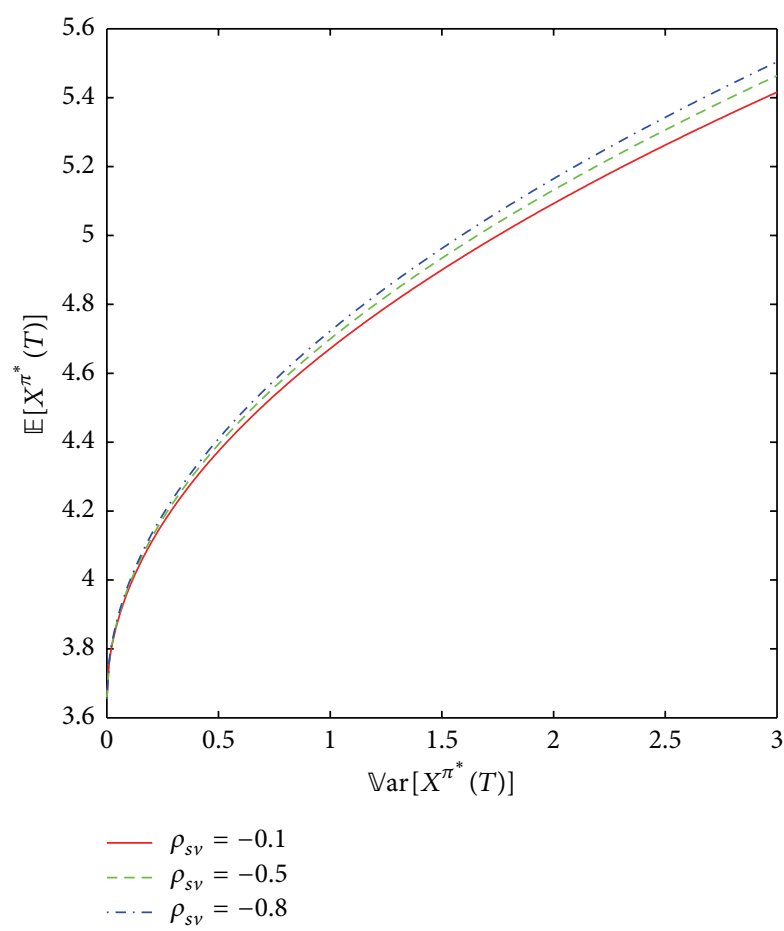

Figure 8: The effect of $\rho_{s v}$ on efficient frontier.

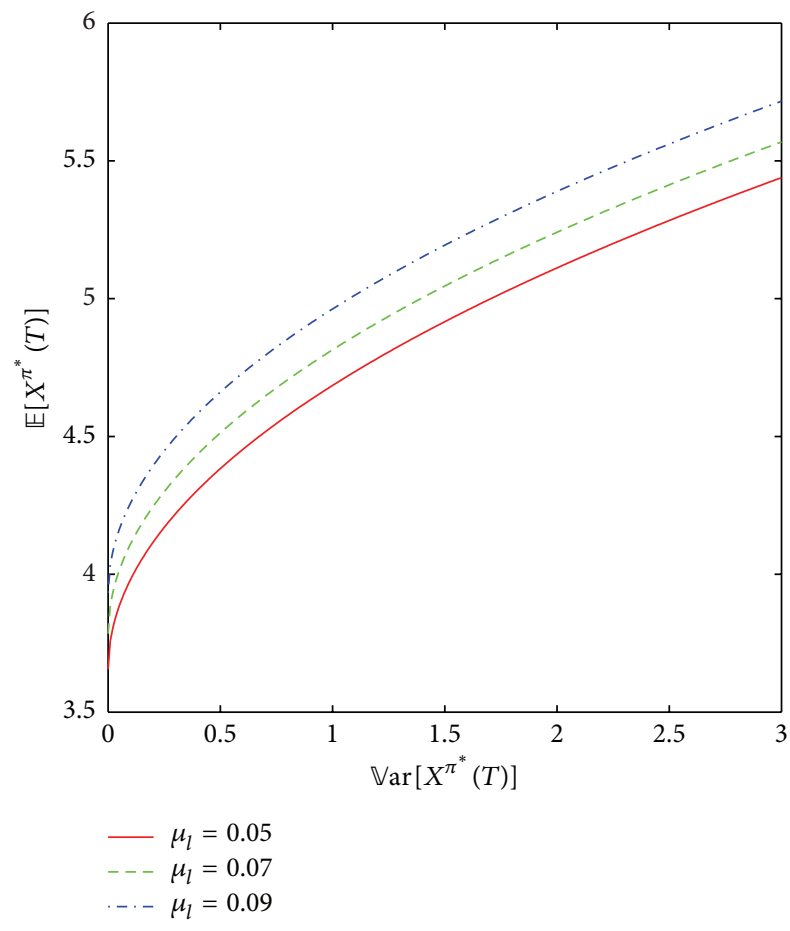

FIgURE 9: The effect of $\mu_{l}$ on efficient frontier.

\section{Conclusion}

In this paper, we investigate the MV portfolio problems for a DC pension plan both before and after retirement. The background risks such as the stochastic volatility of the stock price and the inflation rate are also considered in our model.

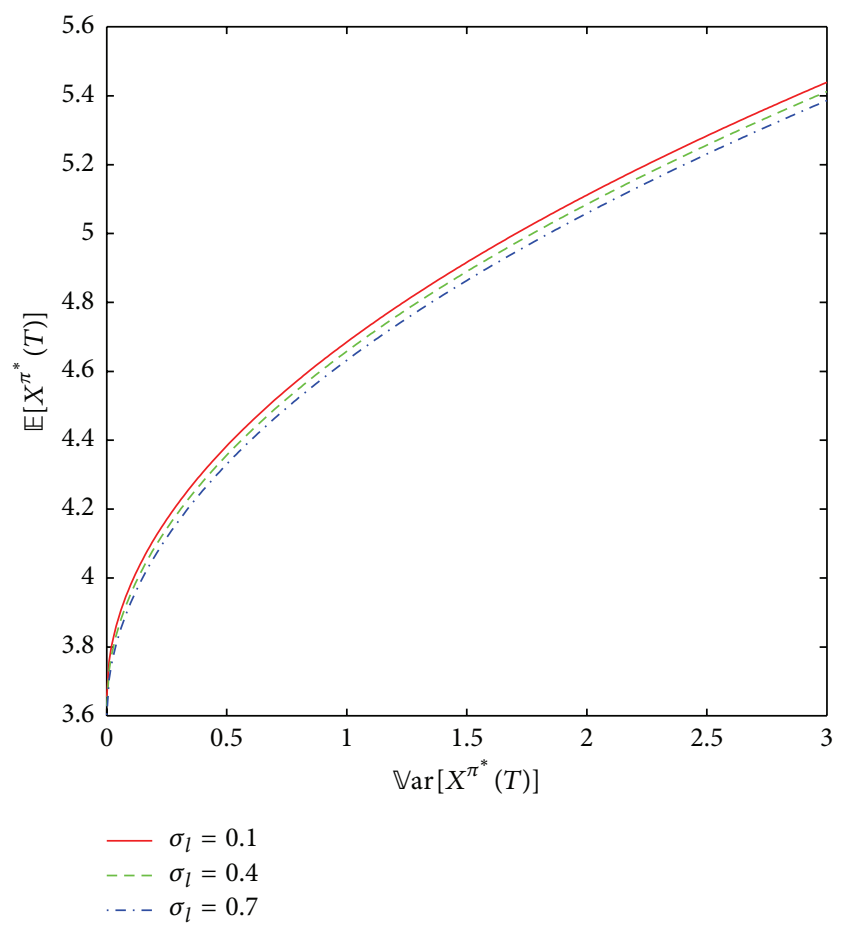

Figure 10: The effect of $\sigma_{l}$ on efficient frontier.

We assume that the pension fund that can be invested in the financial market consists of an inflation-linked bond, a stock, and money market account. Under the framework of game theory, we obtain the equilibrium strategies and the corresponding equilibrium efficient frontiers for both periods. Finally, using Monte Carlo method, we give some numerical analysis and find some interesting results. To obtain the closed-form solution, we assume that the risk aversion parameter of the manager is a constant in this paper. In fact, it may depend on the current wealth or investors' current investment return (see [35]) in practice. In addition, in the period of after retirement, the investment time horizon is assumed to be fixed in our paper. However, since the lifetime of the pension plan member is stochastic, the portfolio problem with uncertain investment time horizon may be more realistic and reasonable, and these problems will be studied in the future.

\section{Appendix}

To prove Theorem 7, first we give a lemma.

Lemma A.1. For the processes $X^{\pi}(t), L(t)$, and $V(t)$ defined in Section 2, if $\phi(t, x, v, l) \in D_{p}(\mathscr{G})$, then $\forall(t, x, v, l) \in \mathscr{G}$ and $\forall \pi \in \Pi(t, x, v, l)$, the following integrals are martingales:

$$
\begin{aligned}
& M_{1}^{\pi}(t, x, v, l)=\int_{t}^{T} \phi_{v}\left(s, X^{\pi}(s), V(s), L(s)\right) \\
& \cdot \sigma_{v} \sqrt{V(s)} d W_{v}(s) ;
\end{aligned}
$$




$$
\begin{aligned}
& M_{2}^{\pi}(t, x, v, l)=\int_{t}^{T} \phi_{x}\left(s, X^{\pi}(s), V(s), L(s)\right) X^{\pi}(s) \\
& \cdot\left(\pi(s) \Sigma-\rho_{p}^{\prime}\right) d W(s) ; \\
& M_{3}^{\pi}(t, x, v, l)=\int_{t}^{T} \phi_{l}\left(s, X^{\pi}(s), V(s), L(s)\right) L(s) \\
& \cdot \beta_{l}^{\prime} d W(s) .
\end{aligned}
$$

Proof. We only prove that $M_{2}^{\pi}(t, x, v, l)$ is martingale, and the others can be proved similarly. Let $(t, x, v, l) \in \mathscr{G}$ and $\pi \in$ $\Pi(t, x, v, l)$. We write $\phi_{x}=\phi_{x}\left(s, X^{\pi}(s), V(s), L(s)\right)$ for short. Note that

$$
\begin{aligned}
& M_{2}^{\pi}(t, x, v, l) \\
& =\int_{t}^{T} \phi_{x} X^{\pi}(s) \bar{\pi}(s) d W_{p}(s) \\
& \quad+\int_{t}^{T} \phi_{x} X^{\pi}(s) \sqrt{V(s)} \pi_{S}(s) d W_{s}(s),
\end{aligned}
$$

where $\bar{\pi}(s)=\left(\pi_{I}(s)-1\right) \sigma_{p}+\pi_{S}(s) \sigma_{s}$. Since $\phi(t, x, v, l) \in$ $D_{p}(\mathscr{G})$, there exist two constants $k_{1}>0$ and $k_{2} \geqslant 1$, such that $\left|\phi_{x}(t, x, v, l)\right| \leqslant k_{1}\left(1+|x|^{k_{2}}+|v|^{k_{2}}+|l|^{k_{2}}\right)$. According to the definition of the admissible strategy, the linear growth, and Yamada and Watanabe conditions satisfied by $V(t)$ and $L(t)$ (see [24] for details), for the first part of (A.2), we have

$$
\begin{aligned}
& \mathbb{E}_{t, x, v, l}\left\{\int_{t}^{T}\left|\phi_{x} X^{\pi}(s) \bar{\pi}(s)\right|^{2} d s\right\} \leqslant \mathbb{E}_{t, x, v, l}\left\{\int _ { t } ^ { T } \left[\left(\phi_{x} X^{\pi}(s)\right)^{4}\right.\right. \\
& \left.\left.+\bar{\pi}(s)^{4}\right] d s\right\} \leqslant \mathbb{E}_{t, x, v, l}\left\{\int_{t}^{T}\left[\phi_{x}^{8}+X^{\pi}(s)^{8}+\bar{\pi}(s)^{4}\right] d s\right\} \\
& \leqslant \mathbb{E}_{t, x, v, l}\left\{\int _ { t } ^ { T } \left[k_{1}^{8}\left(1+\left|X^{\pi}(s)\right|^{k_{2}}+|V(s)|^{k_{2}}+|L(s)|^{k_{2}}\right)^{8}\right.\right. \\
& \left.\left.+X^{\pi}(s)^{8}+\bar{\pi}(s)^{4}\right] d s\right\}<\infty .
\end{aligned}
$$

Furthermore, for the second part of (A.2), we have

$$
\begin{aligned}
& \mathbb{E}_{t, x, v, l}\left\{\int_{t}^{T}\left|\phi_{x} X^{\pi}(s) \sqrt{V(s)} \pi_{S}(s)\right|^{2} d s\right\} \\
& \leqslant \mathbb{E}_{t, x, v, l}\left\{\int_{t}^{T}\left[\left(\phi_{x} X^{\pi}(s) \sqrt{V(s)}\right)^{4}+\pi_{S}(s)^{4}\right] d s\right\} \\
& \leqslant \mathbb{E}_{t, x, v, l}\left\{\int _ { t } ^ { T } \left[\phi_{x}^{8}+\left(X^{\pi}(s) \sqrt{V(s)}\right)^{8}\right.\right. \\
& \left.\left.+\pi_{S}(s)^{4}\right] d s\right\} \leqslant \mathbb{E}_{t, x, v, l}\left\{\int _ { t } ^ { T } \left[\phi_{x}^{8}+X^{\pi}(s)^{16}\right.\right. \\
& \left.\left.+|V(s)|^{8}+\pi_{S}(s)^{4}\right] d s\right\}<\infty .
\end{aligned}
$$

By (A.3) and (A.4), we know that $M_{2}^{\pi}(t, x, v, l)$ is martingale.
Proof of Theorem 7. The proof consists of five steps.

Step 1. Consider an admissible strategy $\pi \in \Pi(t, x, v, l)$. We will claim that if there exists a real value function $Y(t, x, v, l) \in$ $D_{p}(\mathscr{G})$ such that $\forall(t, x, v, l) \in \mathscr{G}$

$$
\begin{array}{r}
\mathscr{A}^{\pi} Y(t, x, v, l)=0, \\
Y(T, x, v, l)=x,
\end{array}
$$

then $Y(t, x, v, l)=y^{\pi}(t, x, v, l)$.

In fact, if we write $Y^{\pi}(t)=Y\left(t, X^{\pi}(t), V(t), L(t)\right)$ for short, by Itô's lemma, we have

$$
\begin{aligned}
& Y^{\pi}(t)=Y^{\pi}(T)-\int_{t}^{T} \mathscr{A}^{\pi} Y^{\pi}(s) d s-\int_{t}^{T} Y_{v}^{\pi}(s) \\
& \cdot \sigma_{v} \sqrt{V(s)} d W_{v}(s) \\
& -\int_{t}^{T}\left[Y_{x}^{\pi}(s) X^{\pi}(s)\left(\pi(s) \Sigma(s)-\rho_{p}^{\prime}\right)\right. \\
& \left.+Y_{l}^{\pi}(s) L(s) \beta_{l}^{\prime}\right] d W(s) .
\end{aligned}
$$

Using Lemma A.1 and taking the conditional expectation on both sides of (A.6), we have

$$
\begin{aligned}
Y(t, x, v, l) & =\mathbb{E}_{t, x, v, l}\left[Y^{\pi}(T)\right]=\mathbb{E}_{t, x, v, l}\left[X^{\pi}(T)\right] \\
& =y^{\pi}(t, x, v, l) .
\end{aligned}
$$

Similarly, by replacing $Y$ and $y$ by $Z$ and $z$, respectively, we can claim that if there exists a real value function $Z(t, x, v, l) \in$ $D_{p}(\mathscr{G})$ such that, $\forall(t, x, v, l) \in \mathscr{G}$,

$$
\begin{aligned}
\mathscr{A}^{\pi} Z(t, x, v, l) & =0, \\
Z(T, x, v, l) & =x^{2},
\end{aligned}
$$

then $Z(t, x, v, l)=z^{\pi}(t, x, v, l)$. We denote $Z^{\pi}(t)=Z\left(t, X^{\pi}(t)\right.$, $V(t), L(t))$ for short.

Step 2. Next, we denote two functions $f^{\pi}(t)=f\left(t, X^{\pi}(t)\right.$, $\left.V(t), L(t), Y^{\pi}(t), Z^{\pi}(t)\right)$ and $F^{\pi}(t)=F\left(t, X^{\pi}(t), V(t), L(t)\right)$ and derive an expression for $f^{\pi}(T)$.

Note that $f^{\pi}(t) \in C^{1,2,2,2,2,2}\left([0, T] \times \mathbb{R}^{5}\right)$ and its all first-order partial derivatives satisfy the polynomial growth condition. By Itô's lemma, we have

$$
\begin{aligned}
& f^{\pi}(T)=f^{\pi}(t)+\int_{t}^{T} d f^{\pi}(s)=f^{\pi}(t) \\
& +\int_{t}^{T}\left[f_{y}^{\pi}(s) \mathscr{A}^{\pi} Y^{\pi}(s)+f_{z}^{\pi}(s) \mathscr{A}^{\pi} Z^{\pi}(s)\right. \\
& \left.+\xi^{\pi}(s)\right] d s+\int_{t}^{T}\left[f_{x}^{\pi}(s)+f_{y}^{\pi}(s) Y_{x}^{\pi}(s)\right. \\
& \left.+f_{z}^{\pi}(s) Z_{x}^{\pi}(s)\right] X^{\pi}(s)(\pi(s) \Sigma(s) \\
& \left.-\rho_{p}^{\prime}\right) d W(s)+\int_{t}^{T}\left[f_{l}^{\pi}(s)\right.
\end{aligned}
$$




$$
\begin{aligned}
& \left.+f_{y}^{\pi}(s) Y_{l}^{\pi}(s)+f_{z}^{\pi}(s) Z_{l}^{\pi}(s)\right] L(s) \beta_{l}^{\prime} d W(s) \\
& +\int_{t}^{T}\left[f_{v}^{\pi}(s)+f_{y}^{\pi}(s) Y_{v}^{\pi}(s)+f_{z}^{\pi}(s) Z_{v}^{\pi}(s)\right] \\
& \cdot \sigma_{v} \sqrt{V(s)} d W_{v}(s) .
\end{aligned}
$$

Substituting (A.5) and (A.8) into (A.9), we obtain

$$
\begin{aligned}
& f^{\pi}(T)=f^{\pi}(t)+\int_{t}^{T} \xi^{\pi}(s) d s \\
& +\int_{t}^{T}\left[f_{l}^{\pi}(s)+f_{y}^{\pi}(s) Y_{l}^{\pi}(s)+f_{z}^{\pi}(s) Z_{l}^{\pi}(s)\right] \\
& \cdot L(s) \beta_{l}^{\prime} d W(s) \\
& +\int_{t}^{T}\left[f_{x}^{\pi}(s)+f_{y}^{\pi}(s) Y_{x}^{\pi}(s)+f_{z}^{\pi}(s) Z_{x}^{\pi}(s)\right] \\
& \cdot X^{\pi}(s)\left(\pi(s) \Sigma(s)-\rho_{p}^{\prime}\right) d W(s) \\
& +\int_{t}^{T}\left[f_{v}^{\pi}(s)+f_{y}^{\pi}(s) Y_{v}^{\pi}(s)+f_{z}^{\pi}(s) Z_{v}^{\pi}(s)\right] \\
& \cdot \sigma_{v} \sqrt{V(s)} d W_{v}(s) .
\end{aligned}
$$

Step 3. Now we show that, $\forall(t, x, v, l) \in \mathscr{G}$,

$$
\begin{aligned}
F & (t, x, v, l) \\
& \geqslant \sup _{\pi \in \Pi(t, x, v, l)} f\left(t, x, v, l, y^{\pi}(t, x, v, l), z^{\pi}(t, x, v, l)\right) .
\end{aligned}
$$

Using Itô's lemma, we derive

$$
\begin{aligned}
& F^{\pi}(t)=F^{\pi}(T)-\int_{t}^{T} d F^{\pi}(s)=F^{\pi}(T) \\
& -\int_{t}^{T} \mathscr{A}^{\pi} F^{\pi}(s) d s-\int_{t}^{T} F_{v}^{\pi}(s) \\
& \cdot \sigma_{v} \sqrt{V(s)} d W_{v}(s) \\
& -\int_{t}^{T}\left[F_{x}^{\pi}(s) X^{\pi}(s)\left(\pi(s) \Sigma(s)-\rho_{p}^{\prime}\right)\right. \\
& \left.+F_{l}^{\pi}(s) L(s) \beta_{l}^{\prime}\right] d W(s) .
\end{aligned}
$$

Since $\mathscr{A}^{\pi} F(t, x, v, l) \leqslant \xi^{\pi}(t, x, v, l)$, we have

$$
\begin{gathered}
F^{\pi}(t) \geqslant F^{\pi}(T)-\int_{t}^{T} \xi^{\pi}(s) d s-\int_{t}^{T} F_{v}^{\pi}(s) \\
\cdot \sigma_{v} \sqrt{V(s)} d W_{v}(s) \\
-\int_{t}^{T}\left[F_{x}^{\pi}(s) X^{\pi}(s)\left(\pi(s) \Sigma(s)-\rho_{p}^{\prime}\right)\right. \\
\left.+F_{l}^{\pi}(s) L(s) \beta_{l}^{\prime}\right] d W(s) .
\end{gathered}
$$

In addition, according to the condition of Theorem $7, F^{\pi}(T)=$ $f^{\pi}(T)$. Thus, inserting (A.10) into (A.13), we get

$$
\begin{aligned}
& F^{\pi}(t) \geqslant f^{\pi}(t)+\int_{t}^{T}\left[f_{l}^{\pi}(s)+f_{y}^{\pi}(s) Y_{l}^{\pi}(s)\right. \\
& \left.+f_{z}^{\pi}(s) Z_{l}^{\pi}(s)-F_{l}^{\pi}(s)\right] L(s) \beta_{l}^{\prime} d W(s) \\
& +\int_{t}^{T}\left[f_{x}^{\pi}(s)+f_{y}^{\pi}(s) Y_{x}^{\pi}(s)+f_{z}^{\pi}(s) Z_{x}^{\pi}(s)\right. \\
& \left.-F_{x}^{\pi}(s)\right] X^{\pi}(s)\left(\pi(s) \Sigma(s)-\rho_{p}^{\prime}\right) d W(s) \\
& +\int_{t}^{T}\left[f_{v}^{\pi}(s)+f_{y}^{\pi}(s) Y_{v}^{\pi}(s)+f_{z}^{\pi}(s) Z_{v}^{\pi}(s)\right. \\
& \left.-F_{v}^{\pi}(s)\right] \sigma_{v} \sqrt{V(s)} d W_{v}(s) .
\end{aligned}
$$

Using Lemma A.1, taking conditional expectation on both sides of (A.14), we obtain

$$
\begin{aligned}
& F(t, x, v, l) \\
& \quad \geqslant \sup _{\pi \in \Pi(t, x, v, l)} f\left(t, x, v, l, y^{\pi}(t, x, v, l), z^{\pi}(t, x, v, l)\right) .
\end{aligned}
$$

Step 4. Consider a special admissible strategy $\pi^{*}$.

(1) According to the result of Step $1, G(t, x, v, l)$ and $H(t, x, v, l)$ satisfy

$$
\begin{aligned}
& G(t, x, v, l)=y^{\pi^{*}}(t, x, v, l), \\
& H(t, x, v, l)=z^{\pi^{*}}(t, x, v, l)
\end{aligned}
$$

(2) If $\pi^{*}$ is a special strategy that makes inequation (A.14) as equation, then we have

$$
\begin{aligned}
& F^{\pi^{*}}(t)=f^{\pi^{*}}(t)+\int_{t}^{T}\left[f_{l}^{\pi^{*}}(s)+f_{y}^{\pi^{*}}(s) Y_{l}^{\pi}(s)\right. \\
& \left.+f_{z}^{\pi^{*}}(s) Z_{l}^{\pi^{*}}(s)-F_{l}^{\pi^{*}}(s)\right] L(s) \beta_{l}^{\prime} d W(s) \\
& +\int_{t}^{T}\left[f_{x}^{\pi^{*}}(s)+f_{y}^{\pi^{*}}(s) Y_{x}^{\pi^{*}}(s)+f_{z}^{\pi^{*}}(s) Z_{x}^{\pi^{*}}(s)\right. \\
& \left.-F_{x}^{\pi^{*}}(s)\right] X^{\pi^{*}}(s)\left(\pi^{* \prime}(s) \Sigma-\rho_{p}^{\prime}\right) d W(s) \\
& +\int_{t}^{T}\left[f_{v}^{\pi^{*}}(s)+f_{y}^{\pi^{*}}(s) Y_{v}^{\pi^{*}}+f_{z}^{\pi^{*}}(s) Z_{v}^{\pi^{*}}\right. \\
& \left.-F_{v}^{\pi^{*}}(s)\right] \sigma_{v} \sqrt{V(s)} d W_{v}(s) .
\end{aligned}
$$

By Lemma A.1, and taking conditional expectation on both sides of (A.17), we have

$$
\begin{aligned}
F & (t, x, v, l) \\
& =f\left(t, x, v, l, y^{\pi^{*}}(t, x, v, l), z^{\pi^{*}}(t, x, v, l)\right) \\
& \leqslant \sup _{\pi \in \Pi(t, x, v, l)} f\left(t, x, v, l, y^{\pi}(t, x, v, l), z^{\pi}(t, x, v, l)\right) .
\end{aligned}
$$


Combining with (A.15) and (A.18), we have

$$
\begin{aligned}
& F(t, x, v, l) \\
& \quad=\sup _{\pi \in \Pi(t, x, v, l)} f\left(t, x, v, l, y^{\pi}(t, x, v, l), z^{\pi}(t, x, v, l)\right) .
\end{aligned}
$$

This means that $\pi^{*}$ is the optimal strategy.

Step 5. Prove that $\pi^{*}$ is an equilibrium strategy.

For any $\pi_{\tau}$ defined in Definition 6 , we replace $\pi$ with $\pi_{\tau}$ in (A.10). Then

$$
\begin{aligned}
& f^{\pi_{\tau}}(t+\tau)=f^{\pi_{\tau}}(t)+\int_{t}^{t+\tau} \xi^{\pi_{\tau}}(s) d s \\
& \quad+\int_{t}^{t+\tau}\left[f_{x}^{\pi_{\tau}}(s)+f_{y}^{\pi_{\tau}}(s) Y_{x}^{\pi_{\tau}}(s)\right. \\
& \left.\quad+f_{z}^{\pi_{\tau}}(s) Z_{x}^{\pi_{\tau}}(s)\right]\left(\pi_{\tau}(s) \Sigma-\rho_{p}^{\prime}\right) d W(s) \\
& \quad+\int_{t}^{t+\tau}\left[f_{l}^{\pi_{\tau}}(s)+f_{y}^{\pi_{\tau}}(s) Y_{l}^{\pi_{\tau}}(s) L(s)\right. \\
& \left.\quad+f_{z}^{\pi_{\tau}}(s) Z_{l}^{\pi_{\tau}}(s) L(s)\right] B_{l}^{\prime} d W(s)+\int_{t}^{t+\tau}\left[f_{v}^{\pi_{\tau}}(s)\right. \\
& \left.\quad+f_{y}^{\pi_{\tau}}(s) Y_{v}^{\pi_{\tau}}(s)+f_{z}^{\pi_{\tau}}(s) Z_{v}^{\pi_{\tau}}(s)\right] \\
& \quad \cdot \sigma_{v} \sqrt{V(s)} d W_{v}(s) .
\end{aligned}
$$

When we replace $T=t+\tau$ and $\pi=\pi^{*}$ in (A.13), we have

$$
\begin{aligned}
& F^{\pi^{*}}(t) \geqslant F^{\pi^{*}}(t+\tau)-\int_{t}^{t+\tau} \xi^{\pi^{*}}(s) d s-\int_{t}^{t+\tau} F_{v}^{\pi^{*}}(s) \\
& \cdot \sigma_{v} \sqrt{V(s)} d W_{v}(s) \\
& -\int_{t}^{t+\tau}\left[F_{x}^{\pi^{*}}(s) X^{\pi^{*}}(s)\left(\pi^{*}(s) \Sigma-\rho_{p}^{\prime}\right)\right. \\
& \left.+F_{l}^{\pi^{*}}(s) L(s) \beta_{l}^{\prime}\right] d W(s) .
\end{aligned}
$$

By the definition of $\pi_{\tau}$, we have $f^{\pi_{\tau}}(t+\tau)=F^{\pi^{*}}(t+\tau)$. Replacing $F^{\pi^{*}}(t+\tau)$ with $f^{\pi_{\tau}}(t+\tau)$ in (A.21), we obtain

$$
\begin{aligned}
& F^{\pi^{*}}(t) \geqslant f^{\pi_{\tau}}(t)+\int_{t}^{t+\tau} \xi^{\pi_{\tau}}(s) d s-\int_{t}^{t+\tau} \xi^{\pi^{*}}(s) d s \\
& -\int_{t}^{t+\tau} F_{x}^{\pi^{*}}(s) X^{\pi^{*}}(s)\left(\pi^{*}(s) \Sigma(s)\right. \\
& \left.-\rho_{p}^{\prime}\right) d W(s)+\int_{t}^{t+\tau}\left[f_{x}^{\pi_{\tau}}(s)\right. \\
& \left.+f_{y}^{\pi_{\tau}}(s) Y_{x}^{\pi_{\tau}}(s)+f_{z}^{\pi_{\tau}}(s) Z_{x}^{\pi_{\tau}}(s)\right]\left(\pi_{\tau}(s) \Sigma(s)\right. \\
& \left.-\rho_{p}^{\prime}\right) d W(s)+\int_{t}^{t+\tau}\left[f_{l}^{\pi_{\tau}}(s)\right.
\end{aligned}
$$

$$
\begin{aligned}
& +f_{y}^{\pi_{\tau}}(s) Y_{l}^{\pi_{\tau}}(s) L(s)+f_{z}^{\pi_{\tau}}(s) Z_{l}^{\pi_{\tau}}(s) L(s) \\
& \left.-F_{l}^{\pi^{*}}(s) L(s)\right] \beta_{l}^{\prime} d W(t)+\int_{t}^{t+\tau}\left[f_{v}^{\pi_{\tau}}(s)\right. \\
& \left.+f_{y}^{\pi_{\tau}}(s) Y_{v}^{\pi_{\tau}}(s)+f_{z}^{\pi_{\tau}}(s) Z_{v}^{\pi_{\tau}}(s)-F_{v}^{\pi^{*}}(s)\right] \\
& \cdot \sigma_{v} \sqrt{V(s)} d W_{v}(s) .
\end{aligned}
$$

Since $F^{\pi^{*}}(t)=f^{\pi^{*}}(t)$ and taking conditional expectation on both sides of (A.22) we obtain

$$
\begin{aligned}
& f^{\pi^{*}}(t) \\
& \quad \geqslant f^{\pi_{\tau}}(t) \\
& \quad+\mathbb{E}_{t, x, v, l}\left[\int_{t}^{t+\tau} \xi^{\pi_{\tau}}(s) d s-\int_{t}^{t+\tau} \xi^{\pi^{*}}(s) d s\right],
\end{aligned}
$$

and then

$$
\lim _{\tau \rightarrow 0} \inf \frac{f^{\pi^{*}}(t)-f^{\pi_{\tau}}(t)}{\tau} \geqslant 0 .
$$

\section{Competing Interests}

The authors declare that there are no competing interests regarding the publication of this paper.

\section{Acknowledgments}

This research is supported by grants of the National Natural Science Foundation of China (nos. 71231008 and 71501176), the Project Funded by China Postdoctoral Science Foundation (2015M580141), Natural Science Foundation of Guangdong Province of China (no. 2014A030312003), and the Foundation of City Development Academy of Gansu Province of China (no. 2014-GSCFY-KJ02).

\section{References}

[1] E. Vigna and S. Haberman, "Optimal investment strategy for defined contribution pension schemes," Insurance: Mathematics and Economics, vol. 28, no. 2, pp. 233-262, 2001.

[2] P. Devolder, M. B. Princep, and I. D. Fabian, "Stochastic optimal control of annuity contracts," Insurance: Mathematics and Economics, vol. 33, no. 2, pp. 227-238, 2003.

[3] P. Battocchio and F. Menoncin, "Optimal pension management in a stochastic framework," Insurance: Mathematics and Economics, vol. 34, no. 1, pp. 79-95, 2004.

[4] J. W. Gao, "Stochastic optimal control of DC pension funds," Insurance: Mathematics and Economics, vol. 42, no. 3, pp. 11591164, 2008.

[5] N.-W. Han and M.-W. Hung, "Optimal asset allocation for DC pension plans under inflation," Insurance: Mathematics \& Economics, vol. 51, no. 1, pp. 172-181, 2012. 
[6] G. H. Guan and Z. X. Liang, "Optimal management of DC pension plan in a stochastic interest rate and stochastic volatility framework," Insurance: Mathematics and Economics, vol. 57, pp. 58-66, 2014.

[7] P. Chen and H. L. Yang, "Pension funding problem with regimeswitching geometric Brownian motion assets and liabilities," Applied Stochastic Models in Business and Industry, vol. 26, no. 2, pp. 125-141, 2010.

[8] D. Li and W.-L. Ng, "Optimal dynamic portfolio selection: multiperiod mean-variance formulation," Mathematical Finance, vol. 10, no. 3, pp. 387-406, 2000.

[9] X. Y. Zhou and D. Li, "Continuous-time mean-variance portfolio selection: a stochastic LQ framework," Applied Mathematics and Optimization, vol. 42, no. 1, pp. 19-33, 2000.

[10] S. Basak and G. Chabakauri, "Dynamic mean-variance asset allocation," Review of Financial Studies, vol. 23, no. 8, pp. 29703016, 2010.

[11] T. Björk and A. Murgoci, "A general theory of Markovian time inconsistent stochastic control problems," Working Paper, Stockholm School of Economics, Stockholm, Sweden, 2010.

[12] T. Björk, A. Murgoci, and X. Y. Zhou, "Mean-variance portfolio optimization with state-dependent risk aversion," Mathematical Finance, vol. 24, no. 1, pp. 1-24, 2014.

[13] L. He and Z. X. Liang, "Optimal investment strategy for the DC plan with the return of premiums clauses in a mean-variance framework," Insurance: Mathematics and Economics, vol. 53, no. 3, pp. 643-649, 2013.

[14] X. Q. Liang, L. H. Bai, and J. Y. Guo, "Optimal time-consistent portfolio and contribution selection for defined benefit pension schemes under mean-variance criterion," The ANZIAM Journal, vol. 56, no. 1, pp. 66-90, 2014.

[15] F. Menoncin and E. Vigna, "Mean-variance target-based optimisation in DC plan with stochastic interest rate," Tech. Rep. 337, Collegio Carlo Alberto, 2013.

[16] E. Vigna, "On efficiency of mean-variance based portfolio selection in defined contribution pension schemes," Quantitative Finance, vol. 14, no. 2, pp. 237-258, 2014.

[17] H. Yao, Z. Yang, and P. Chen, "Markowitz's mean-variance defined contribution pension fund management under inflation: a continuous-time model," Insurance: Mathematics and Economics, vol. 53, no. 3, pp. 851-863, 2013.

[18] A. Zhang and C.-O. Ewald, "Optimal investment for a pension fund under inflation risk," Mathematical Methods of Operations Research, vol. 71, no. 2, pp. 353-369, 2010.

[19] H. L. Wu, L. Zhang, and H. Chen, "Nash equilibrium strategies for a defined contribution pension management," Insurance: Mathematics and Economics, vol. 62, pp. 202-214, 2015.

[20] J. C. Cox, "The constant elasticity of variance option pricing model," The Journal of Portfolio Management, vol. 23, pp. 15-17, 1996.

[21] S. L. Heston, "A closed-form solution for options with stochastic volatility with applications to bond and currency options," Review of Financial Studies, vol. 6, no. 2, pp. 327-343, 1993.

[22] E. M. Stein and J. C. Stein, "Stock price distributions with stochastic volatility: an analytic approach," Review of Financial Studies, vol. 4, no. 4, pp. 727-752, 1991.

[23] X. Lin and Y. Li, "Optimal reinsurance and investment for a jump diffusion risk process under the CEV model," North American Actuarial Journal, vol. 15, no. 3, pp. 417-431, 2011.

[24] Z. Li, Y. Zeng, and Y. Lai, "Optimal time-consistent investment and reinsurance strategies for insurers under Heston's SV model," Insurance: Mathematics and Economics, vol. 51, no. 1, pp. 191-203, 2012.

[25] Y. Shen and Y. Zeng, "Optimal investment-reinsurance strategy for mean-variance insurers with square-root factor process," Insurance: Mathematics and Economics, vol. 62, pp. 118-137, 2015.

[26] J. W. Gao, "Optimal portfolios for DC pension plans under a CEV model," Insurance: Mathematics \& Economics, vol. 44, no. 3, pp. 479-490, 2009.

[27] J. W. Xiao, Z. Hong, and C. L. Qin, "The constant elasticity of variance $(\mathrm{CEV})$ model and the Legendre transform-dual solution for annuity contracts," Insurance: Mathematics and Economics, vol. 40, no. 2, pp. 302-310, 2007.

[28] M. J. Brennan and Y. Xia, "Dynamic asset allocation under inflation," Journal of Finance, vol. 57, no. 3, pp. 1201-1238, 2002.

[29] M. Kwak and B. H. Lim, "Optimal portfolio selection with life insurance under inflation risk," Journal of Banking and Finance, vol. 46, no. 1, pp. 59-71, 2014.

[30] A. Anari and J. Kolari, "Stock prices and inflation," Journal of Financial Research, vol. 24, no. 4, pp. 587-602, 2001.

[31] B. S. Lee, "Stock returns and inflation revisited: an evaluation of the inflation illusion hypothesis," Journal of Banking and Finance, vol. 34, no. 6, pp. 1257-1273, 2010.

[32] J. Liu and J. Pan, "Dynamic derivative strategies," Journal of Financial Economics, vol. 69, no. 3, pp. 401-430, 2003.

[33] R. Gerrard, S. Haberman, and E. Vigna, "Optimal investment choices post-retirement in a defined contribution pension scheme," Insurance: Mathematics and Economics, vol. 35, no. 2, pp. 321-342, 2004.

[34] M. Di Giacinto, S. Federico, F. Gozzi, and E. Vigna, "Income drawdown option with minimum guarantee," European Journal of Operational Research, vol. 234, no. 3, pp. 610-624, 2014.

[35] F. H. Wen, Z. F. He, Z. F. Dai, and X. G. Yang, "Characteristics of investors' risk preference for stock markets," Economic Computation and Economic Cybernetics Studies and Research, vol. 48, no. 3, pp. 235-254, 2014. 


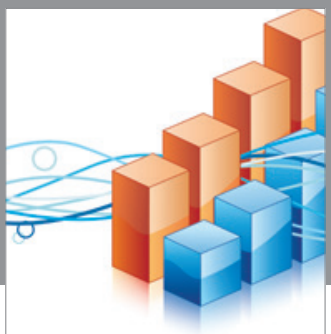

Advances in

Operations Research

vatem alat4

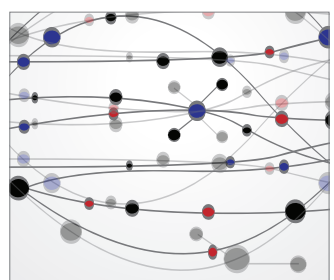

\section{The Scientific} World Journal
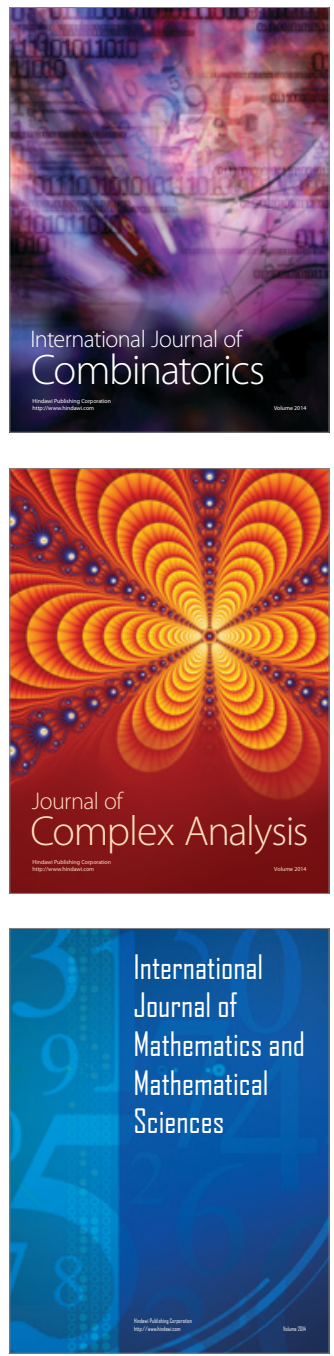
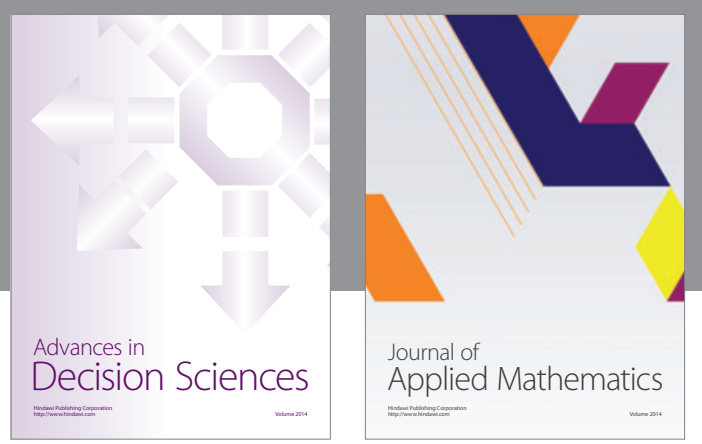

Algebra

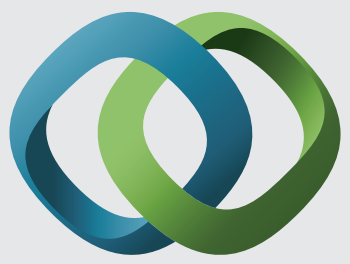

\section{Hindawi}

Submit your manuscripts at

http://www.hindawi.com
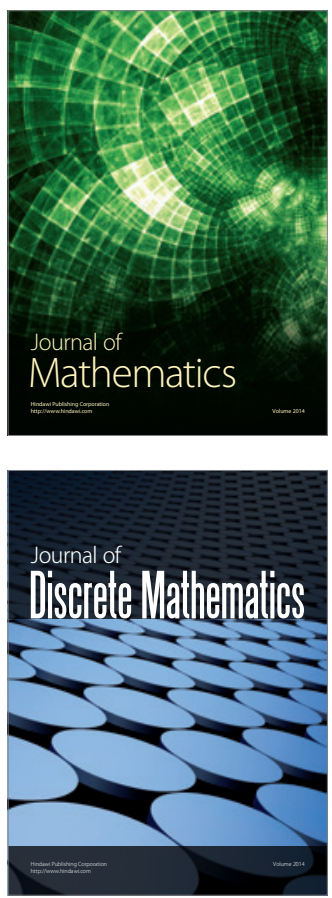

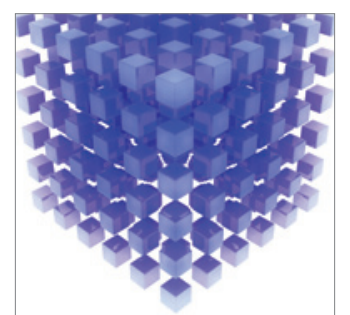

Mathematical Problems in Engineering
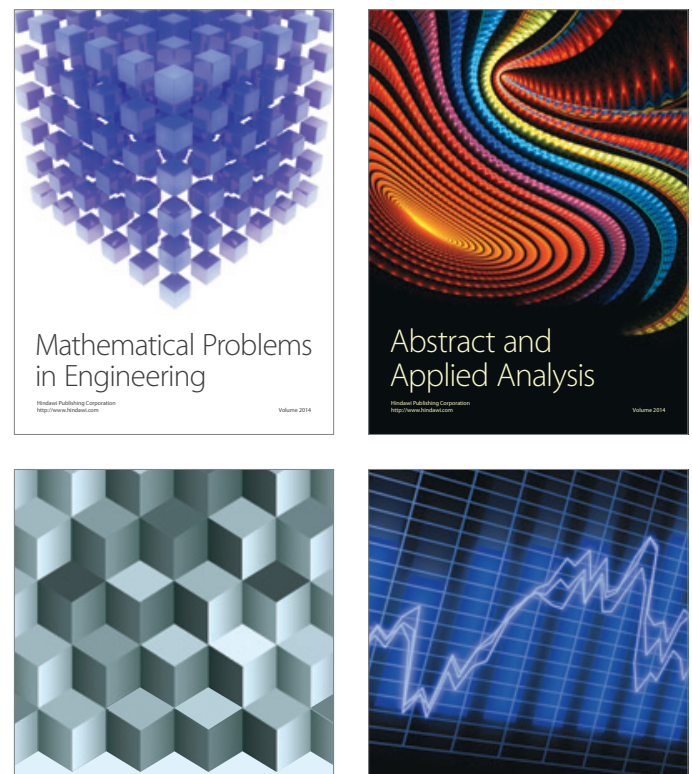

Journal of

Function Spaces

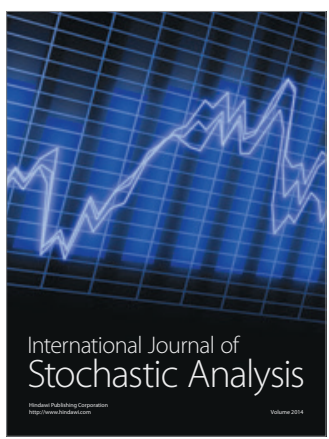

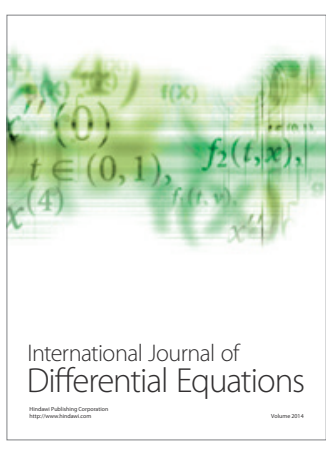
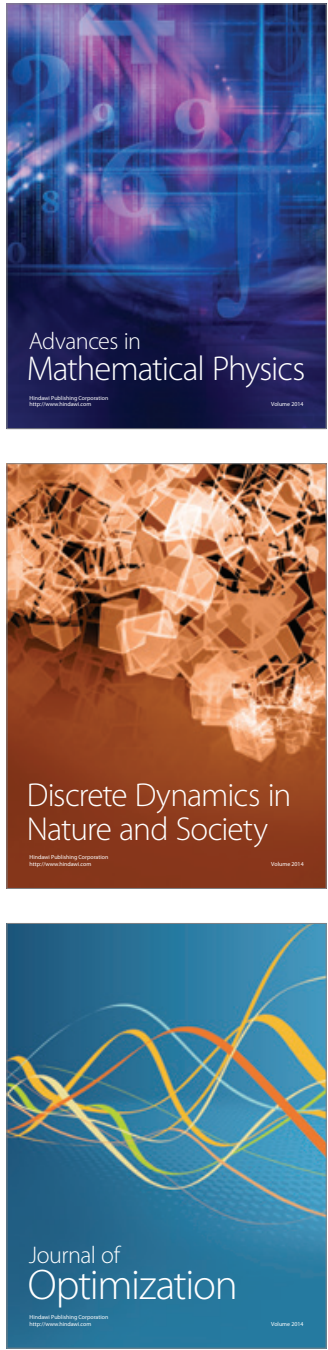